\title{
1 Developmental regulators FlbE/D orchestrate the polarity 2 site-to-nucleus dynamics of the fungal bZIP FlbB
}

3 Ainara Otamendi ${ }^{1}$, Elixabet Perez-de-Nanclares-Arregi ${ }^{1}$, Elixabet Oiartzabal ${ }^{1}$, Marc S.

4 Cortese $^{1}$, Eduardo A. Espeso ${ }^{2}$, Oier Etxebeste ${ }^{1, *}$.

$7{ }^{1}$ Biochemistry II laboratory, Department of Applied Chemistry, Faculty of Chemistry,

8 University of The Basque Country, 20018 San Sebastian, Spain.

$9{ }^{2}$ Department of Cellular and Molecular Biology, Centro de Investigaciones Biológicas

10 (CSIC), Ramiro de Maeztu 9, 28040 Madrid, Spain.

$11 *$ Corresponding author: Oier Etxebeste; E-mail: oier.echeveste@ehu.eus; Tel: (+34)

12943 018517; Fax: (+34) 943 015270; Biochemistry II laboratory, Dept. of Applied

13 Chemistry, Faculty of Chemistry, University of the Basque Country, Manuel de

14 Lardizabal, 3, 20018, San Sebastian.

16 Running title: Spatio-temporal control of FlbB dynamics

Keywords: filamentous fungi, hyphal growth, development, signal transduction,

19 polarity site-to-nucleus communication 


\section{Abstract (175 words).}

2 Permanently polarized cells have developed transduction mechanisms linking

3 polarity-sites with gene regulation in the nucleus. In neurons, one mechanism is

4 based on long-distance retrograde migration of transcription factors (TFs).

5 Aspergillus nidulans FlbB is the only known fungal TF shown to migrate

6 retrogradely to nuclei from the polarized region of fungal cells known as hyphae.

7 There, FlbB controls developmental transitions by triggering the production of

8 asexual multicellular structures. FlbB dynamics in hyphae is orchestrated by

9 regulators FlbE and FlbD. At least three FlbE domains are involved in the

10 acropetal transport of FlbB, with a final MyoE/actin filament-dependent step from

11 the subapex to the apex. Experiments employing a T2A viral peptide-containing

12 chimera (FlbE::mRFP::T2A::FlbB::GFP) suggest that apical FlbB/FlbE

13 interaction is inhibited in order to initiate a dynein-dependent FlbB transport to

14 nuclei. FlbD controls the nuclear accumulation of FlbB through a cMyb domain

15 and a C-terminal LxxLL motif. Overall, results elucidate a highly dynamic pattern

16 of FlbB interactions, which enable timely developmental induction. Furthermore,

17 this system establishes a reference for TF-based long-distance signaling in

18 permanently polarized cells. 
1

3 living organisms. With this aim, eukaryotic cells monitor the environment, receive

4 signals from it, interiorize, amplify and integrate these cues, and finally convey the

5 corresponding information to nuclei in the form of proteins (transcription factors; TFs)

6 that are able of modifying gene expression patterns and, consequently, induce the

7 adaptive cellular response. All these steps are carried out following a variety of

8 molecular mechanisms that are generally classified as signal transduction pathways [1].

Permanent polarization of specific cell types such as neurons, pollen tubes or hyphae (see next paragraph) significantly increases the distance between polarity sites (i.e. growth cones in neurons or tips of hyphae) and nuclei, complicating the transduction of signals along this path [2-5]. Thus, permanently polarized cells have necessarily developed transduction mechanisms that are capable of covering the corresponding distances with speed and reliability. In neurons, these mechanisms are based mainly on calcium waves, but also on the retrograde transport of macromolecular complexes [5-7]. Retrograde transport of macromolecular complexes are used to control key neuronal processes such as the response to injury [8]. The main messengers in those complexes can be kinases which ultimately transfer the signal to a $\mathrm{TF}$ in the nuclear periphery or inside the nucleus, or TFs themselves that are able to migrate basipetally from the polarity site to the nucleus and directly modify gene expression.

Hyphae are the characteristic cell type of filamentous fungi. These permanentlypolarized structures elongate by pulsed extension of the tip apex [9] that is dependent on receiving plasma membrane and cell-wall materials that are transported first on microtubules (MT) and then on actin filaments [10,11]. The fast polar growth of fungal 
1 hyphae increases turgor pressure impinged on the substrate, facilitating its efficient

2 colonization. Hyphae also sense the environment and vary their growth direction in

3 response to different stimuli such as chemical, topographical or electrical signals [12-

4 17]. Under unfavorable growth conditions [18-20] and/or in response to specific

5 chemical signals [21], developmental transitions are triggered in hyphae, producing

6 sexual or asexual spores depending on the stimulus [22]. Asexual spores are mitotic

7 spores constituting the prevalent mechanism for dissemination of fungi. Recent findings

8 have shown the existence of signaling complexes retrogradely transiting from the tip of

9 hyphae to nuclei [3,5,23-26]. Although a limited number of them have been

10 characterized, these mechanisms are based, as in neurons, either on kinase modules or

11 the direct basipetal transport of TFs. These factors control stress responses as well as the

12 sexual and asexual multicellular developmental cycles of filamentous fungal species

13 such as Aspergillus nidulans.

This ascomycete is the preferential reference organism used in the study of the

15 genetic and molecular control of fungal asexual development [27]. Most of the

16 developmental transitions leading this fungus to the production of asexual spores,

17 known as conidia, are controlled by the central developmental pathway (CDP) [20].

18 brlA is the first CDP gene and, thus, many signal transduction pathways activating or

19 inhibiting conidiation converge at its promoter region so as to coordinately control its

20 expression [28]. The upstream developmental activation (UDA) pathway is one of the

21 main signal transduction pathways controlling brlA expression [29]. Three UDA TFs,

22 FlbB, FlbC and FlbD, bind the $\operatorname{brlA}$ promoter [30,31] and control conidiation jointly

23 with TFs from other pathways [28]. FlbC has been located in a sub-pathway parallel to

24 that defined by FlbB and FlbD [31]. The regulatory activity of FlbB and FlbD is 
1 interdependent, since the former controls the expression of $f l b D$ but it cannot bind the

2 promoter of $\operatorname{brlA}$ in the absence of FlbD [30].

$4 \quad$ FlbB is the first known fungal TF showing an apical localization [32]. Indeed, previous

5 work showed that the FlbE-dependent tip localization of FlbB is a pre-requisite for

6 timely control of $\operatorname{brlA}$ expression and that this TF is transported basipetally from the

7 growth region to nuclei (hyphae of $A$. nidulans are multinucleate) [23]. In this work, we

8 have delimited in space and time the role of UDA proteins FlbE and FlbD in regards to

9 the control of FlbB dynamics. Through at least three domains, FlbE plays an essential

10 role in the acropetal dynamics of FlbB towards the growing apex of the tip.

11 Nevertheless, results suggest that $\mathrm{FlbB} / \mathrm{FlbE}$ interaction is inhibited by an as yet

12 unknown mechanism, initiating a tip-to-nucleus dynein- (and thus MT-) dependent

13 basipetal migration of FlbB. FlbD positively controls the nuclear accumulation of FlbB

14 through at least a highly conserved $\mathrm{N}$-terminal cMyb transcriptional regulatory domain

15 and a C-terminal LxxLL motif. Taking everything into consideration, results suggest

16 that a precise sequence of interactions determines the directionality of FlbB dynamics,

17 facilitating communication between the hyphal tip and nuclei, and consequently leading

18 to timely coordination of the TFs that control the expresson of brlA.

20 Results.

21 Constitutive upregulation of $f l b E$ increases the apical concentration of FlbB and 22 induces conidiation in liquid culture.

24 gradient in nuclei, with the highest concentration found in the most apical nucleus and 
1 steadily decreasing quantities in successive nuclei [32]. Constitutive expression of a

2 GFP::FlbB chimera driven by the $g p d A^{\min i}$ promoter [33] increases the nuclear pool of

3 FlbB, with all nuclei being filled with more or less equal amounts of the TF [23].

4 However, this excess in the nuclear pool of FlbB does not correlate with an increase in

5 conidia production because it corresponds to a transcriptionally inactive form of this TF.

6 The absence of the FlbB-interactor protein FlbE precludes accumulation of FlbB at the

7 tip, linking the activation of FlbB to its transport to and/or accumulation at the growth

8 region as well as the presence of FlbE [23,34].

9 To determine whether the apical concentration of FlbB is directly dependent on

10 the quantity of FlbE available, we constitutively expressed FlbE::mRFP or FlbE::Stag

11 fusions, each driven by the $g p d A^{\text {mini }}$ promoter (integrated at the flbE locus; see Figure

12 EV1A), in a $g p d A^{\text {mini }}:$ GFP::FlbB strain. Both dual over-expression (OE) strains showed

13 a statistically significant increase in the apical fluorescence intensity of GFP::FlbB

14 compared to the parental $g p d A^{\min i}:: \mathrm{GFP}:: F \mathrm{bB}$ strain (Figure 1A). The green

15 fluorescence intensity ratio between the tip and the most apical nucleus increased

16 significantly from $1.11 \pm 0.24$ in the control to $1.78 \pm 0.45$ in the FlbE::Stag strain and

$171.83 \pm 0.53$ in the FlbE::RFP strain $\left(n=15\right.$ hyphae for each strain; $p=6.3 \times 10^{-6}$ and 5.5

$18 \times 10^{-5}$, respectively). These results strongly suggest that the concentration of FlbB at the

19 hyphal apex is tightly linked to the concentration of FlbE.

20 Next, we checked if this higher apical accumulation of FlbB and FlbE was

21 accompanied by higher conidia production and/or premature induction of conidiation

22 (Figure 1B and Figure EV1B). On solid medium, wild-type strains conidiate because

23 hyphae are exposed to the air environment [20]. The three strains produced similar

24 amounts of conidia $(n=3$ for each strain; $\mathrm{p}=0.41$ and 0.59 , when mRFP- or S-tagged

25 strains were compared to the reference strain) (Figure EV1B). Clear differences arose, 
1 however, after 26 hours of culture in liquid medium compared to the following three

2 reference strains (Figure 1B): 1) a strain expressing GFP::FlbB and FlbE::mCherry

3 chimeras, each driven by its respective native promoter; 2) a strain expressing a

$4 \quad g p d A^{\text {mini }}$-driven GFP::FlbB chimera; or 3) a strain expressing a $g p d A^{\text {mini }}$-driven

5 FlbE::RFP chimera. While, as expected, reference strains formed only vegetative

6 hyphae (triangles in Figure 1B), the double-gpdA $A^{\text {mini }}$ strain produced conidiophores (the

7 asexual structures bearing conidia) composed of all the characteristic cell types (asterisk

8 in Figure 1B). Results strongly suggest that the apical accumulation of FlbB is directly

9 dependent on FlbE concentration, and that a higher accumulation of the FlbB/FlbE

10 signaling complex at the tip correlates, under certain growth conditions, with the ability

11 to induce conidiation prematurely.

13 Domain analysis of FlbE.

14 Due to the key role of FlbE in the apical accumulation of FlbB and, 15 consequently, the control of conidiation, we proceeded with a deeper characterization of 16 functional domains in FlbE. Preliminary analysis of the FlbE sequence revealed the 17 presence of two main domains (Figure 1C) [34]. Motif A spans from residue M1 to I93, 18 and motif B resides between residues R127 and P151. Both regions were connected by a 19 non-conserved linker sequence (94-126). The C-terminal region of FlbE, comprising 20 residues from D153 to S202, showed a concentration of acidic, mainly aspartic, residues 21 in the domain between D153 and D175, which are relatively conserved in most 22 orthologs [34,35]. In contrast, the region between G176 and S202 showed no 23 conservation among FlbE orthologs.

24 A more detailed HMM analysis divided motif A into four conserved regions,: E1

25 (1-33), E2 (45-57), E3 (58-73) and E4 (79-90) (domain B, positions 127-151, was 
1 renamed as E5 in this scheme) (Figure 1C) [35]. Specific residues within conserved

2 domains were selected for alanine replacement. Due to its absolute conservation in FlbE

3 orthologs [35], W11 was selected to study the role of domain E1. As a potential

4 ubiquitination target [36], K51 was selected within domain E2. D70 and D73 were also

5 mutated as they might contribute to a polyproline helix structure in domain E3. Y85 and

6 V86, respectively, which are located within a predicted hydrophobic cluster in domain

7 E4 and are highly conserved within FlbE orthologs, were also selected. Finally, and due

8 to the characteristic conformational restrictions imposed by prolines, P182 was selected

9 in the poorly-conserved C-terminal region. We had previously described that mutation

10 S120P within the linker region and an A131V substitution within domain E5 both

11 caused delocalization of FlbE from the tip and, consequently, a fluffy aconidial

12 phenotype [34]. Thus, they were not included in the current analysis.

13 First, a strain expressing a $g p d A^{\operatorname{mini}}$-driven FlbE::GFP chimera was generated

14 following the same procedure as that shown in Figure EV1A. Using its genomic DNA

15 as a template, we proceeded with the site-directed mutagenesis approach described in

16 Figure EV1C, in order to generate strains of $A$. nidulans expressing the $g p d A^{\operatorname{mini}}$-driven

17 mutant FlbE::GFP forms described above. The replacement of the targeted amino acid

18 and accuracy of $f l b E$ sequence were confirmed by sequencing of the complete open

19 reading frame. The phenotype of the strains and the subcellular localization of the

20 mutant chimeras were then analyzed (Figure 1D; Figure 1E for mRFP-tagged mutant

21 FlbE forms; Figure EV1D for GFP-tagged counterparts). Mutations K51A and P182A

22 did not alter conidia production $(\mathrm{p}>0.05$ compared to the parental wild-type strain; $\mathrm{n}=$

233 replicates for each strain) nor FlbB/FlbE localization. The fact that P182A mutation

24 did not inhibit conidia production or FlbE/FlbB localization probably reflects that

25 hypothetic folding induced by this residue is not essential for FlbE functionality. 
2 phenotype, with significantly reduced conidia production compared to the parental

3 strain (Figure 1D). The wild-type $g p d A^{\text {mini }}:$ FlbE::GFP strain produced $1.1 \times 10^{7} \pm 1.2 \mathrm{x}$

$4 \quad 10^{6}$ conidia/ $\mathrm{cm}^{2}$ while W11A, D70A;D73A and Y85A;V86A mutants produced 4.1-6.2

$5 \times 10^{6} \pm 0.9-2.0 \times 10^{6}$ conidia/ $/ \mathrm{cm}^{2}(\mathrm{p}=0.020,0.006$ and 0.010 , respectively, in the three

6 replicate experiments, with $n=3$ replicates for each strain). Additionally, we observed

7 differences in FlbE localization among these mutants. While W11A and Y85A;V86A

8 mutations caused the absence of FlbE from the hyphal tip and a dispersion along the

9 cytoplasm, $g p d A^{\text {mini }}$-driven $\mathrm{FlbE}^{(\mathrm{D} 70 \mathrm{~A} ; \mathrm{D} 73 \mathrm{~A})}: \mathrm{GFP}$ (or -mRFP tagged) still accumulated at

10 the tip (Figure 1E and Figure EV1D). However, due to the fluffy phenotype of the strain,

11 it can be inferred that this apical form of FlbE is not fully functional or is not

12 accumulated at the tip above a hypothetic threshold concentration (Figure 1D). In

13 general, FlbB localization correlated with that of FlbE, being delocalized from the tip in

14 W11A and Y85A;V86A mutants but not completely in the D70A:D73A mutant (Figure

15 1E; note also in Figure EV1E the localization of a GFP::FlbB chimera driven by the

16 native $f l b B$ promoter in a strain co-expressing a $g p d A^{\operatorname{mini}}$-driven $\mathrm{FlbE}^{(\mathrm{D} 70 \mathrm{~A} ; \mathrm{D} 73 \mathrm{~A})}:: \mathrm{mRFP}$

17 chimera). All $g p d A^{\text {mini }}$-driven FlbE::GFP chimeras were detected by immunodetection

18 and showed the same mobility on Western blots (Figure EV1F). against a bacterially expressed GST::FlbB form (Figure 1F). The results correlated with

21 the localization of FlbE/FlbB. Those mutations within FlbE inhibiting the apical

22 localization of FlbE/FlbB also showed inhibition of the in vitro interaction with

23 GST::FlbB while those not affecting apical localization exhibited the interaction. 
1 Domain E1 is essential but insufficient for the apical localization of FlbB and FlbE.

Next, we focused on domain E1 because this region was predicted to be a signal

3 peptide in the AspGD (www.aspgd.org) database based on Interpro [37] search results.

4 As a preliminary approach to investigate its possible function, the wild-type FlbE

5 sequence was tagged with GFP at the N-terminus and expressed driven by the native

$6 f l b E$ promoter (see the tagging procedure in Figure EV2A). N-terminal GFP tagging of

7 FlbE caused an aconidial phenotype that was the consequence of the delocalization of

8 FlbE from the tip (Figure 2A and 2B). As stated before, this result contrasts with the

9 wild-type functionality observed for a C-terminal fluorescent tagged FlbE constructs

10 (Figure 1 and EV1). A similar aconidial phenotype and delocalization of FlbE from the

11 tip were observed in cells of a strain expressing the truncated FlbE ${ }^{(34-201)}:$ :GFP form

12 lacking the putative signal peptide (Figure 2A, 2B and EV2B). These results show the

13 importance of domain $\mathrm{E} 1$ in the function of FlbE in the cell.

Mislocalization of FlbE with a N-terminal GFP tag may be related to failure of any attempt to show an interaction between a bacterially expressed GST::FlbE chimera

16 (used as bait) and FlbB::HA $3 x$ (Figure EV2C). Behavior of this type of construct could

17 be due to interaction between the GFP moiety and parts of the FlbE sequence or its

18 interference with localization motifs. However, an immunoprecipitation experiment

19 performed with FlbE::mRFP shows that it can be used successfully as bait, retaining

20 GFP::FlbB when it is in the wild-type form but not when it bears the W11A mutation

21 within domain E1 (Figure 2C).

In order to determine whether domain E1 is sufficient to target FlbB to the tip of

23 hyphae, three DNA constructs were generated. One of them contained the entire FlbE

24 protein tagged in the C-terminus with an FlbB::GFP chimera (Figure EV2D). Second 
1 and third constructs bore only the putative signal peptide of FlbE (amino acids from 1 to

2 39) fused to FlbB::GFP, but the third one included the mutation W11A (FlbE ${ }^{(1-39 ;}$

$3^{\mathrm{W} 11 \mathrm{~A})}::$ FlbB::GFP) (Figure EV2E). All of them were driven by $g p d A^{\operatorname{mini}}$. Protoplasts of a

$4 \Delta f l b B$ strain were transformed and recombination of DNA constructs was selected at the

5 flbE locus, so as to guarantee that the only source of FlbB and FlbE was the one derived

6 from the translation of the constructs. Correct recombination of the constructs was

7 confirmed by Southern-blot and the correctness of the reading frame in the transition

8 from $f l b E$ to $f l b B$ sequences was confirmed by genomic sequencing. All transformants

9 showed the characteristic aconidial phenotype of the null $f l b E$ strain (Figure 2D).

10 Protein chimeras of the expected size were detected in all strains by immunodetection

11 (Figure EV2F).

12 The fluorescence of the FlbE::FlbB::GFP chimera was detected at the tip of

13 hyphae, suggesting that it can meet all requirement for utilization of the acropetal

14 transport pathway (Figure 2E). Despite the constitutive overexpression provided by the

$15 g p d A^{\operatorname{mini}}$ promoter in this chimera, it was not detected in nuclei ( $\mathrm{n}=45$ hyphae).

16 Considering the fluffy phenotype of the strain, it can be hypothesized that FlbB cannot

17 be released from the tip, inhibiting its basipetal transport and thus the transcriptional

18 control of brlA in nuclei. In contrast, chimeras bearing only domain E1 of FlbE

19 accumulated in cytoplasmic filamentous structures (Figure 2E) which resembled

20 mitochondria [38]. These results showed that domain E1 of FlbE is not sufficient to

21 target FlbB to the tip and highlighted the importance of additional regions of FlbE for

22 apical localization, such as domains E4, E5, the linker region (see above; [34]), and

23 even domain E3.

24 To further investigate the hypothetic requirement of an inhibition of the

25 interaction between FlbB and FlbE in order to initiate basipetal transport of the former, 
1 additional FlbE chimeras were generated and expressed in a $\Delta f l b B$ background.

2 Constructs were integrated in the flbE locus (as above). Transformant strains expressed

3 a $g p d A^{\min i}$-driven chimera consisting of FlbE::mRFP fused to and in frame with

4 FlbB::GFP through a wild-type or a mutant short sequence corresponding to the viral

5 peptide T2A (EGRGSLLTCGDVEENPGP or EGRGSLLTCGDVEENPAP,

6 respectively) (Figure EV2G). During translation of the mRNA, T2A induces the

7 cleavage of the peptide at its last codon (G17-P18) but without blocking translation,

8 which continues until the stop codon of the construct [39]. The maintenance of the

9 correct reading frame was confirmed by sequencing and the synthesis of chimeras of the

10 expected size by immunodetection (Figure EV2H). Peptides with sizes corresponding to

11 FlbE::mRFP and FlbB::GFP were detected in strains expressing the wild-type T2A

12 sequence. However, bands corresponding to the uncleaved, full-length chimera were

13 also detected, as in the case of strains bearing the mutant $\mathrm{T} 2 \mathrm{~A}^{(\mathrm{G} 17 \mathrm{~A})}$ sequence. This

14 suggested that the efficiency of T2A in our system was partial. Accordingly, the strains

15 with the wild-type T2A sequence partially restored conidia production to levels between

16 those of wild-type and aconidial reference strains (Figure 2F). Those results correlated

17 with the subcellular localization of FlbB. When the wild-type T2A peptide was

18 expressed, FlbB recovered nuclear localization but in the form of a weak gradient,

19 despite being expressed driven by $g p d A^{\operatorname{mini}}$ (Figure 2G). In this case, the fluorescence

20 intensity ratio between the tip and the most apical nucleus decreased from $2.78 \pm 0.65$ in

21 strains expressing the mutant $\mathrm{T} 2 \mathrm{~A}^{(\mathrm{G} 17 \mathrm{~A})}$ form (cytoplasmic fluorescence was considered

22 as the value for nuclei) to $1.60 \pm 0.48$ when the wild-type T2A form was expressed ( $\mathrm{p}=$

$230.0000079 ; \mathrm{n}=15$ hyphae for each strain). Overall, results in this section extend our

24 previous model showing that the apical interaction between FlbB and FlbE is in all

25 probability inhibited in order to initiate the basipetal transport of the TF. 
2 Apical accumulation of FlbB and FlbE, but not their interaction, requires cysteines

$3 \quad 272$ and 382 of FlbB.

4 The central and C-terminal domains of FlbB are essential for its accumulation at

5 the tip and the induction of conidiation under standard culture conditions [23]. FlbB

6 contains six Cys residues within these domains: Cys236, 272, 280, 303, 382 and 397

7 (Figure 3A). These Cys residues show higher or lower conservation within FlbB

8 orthologs [35]. Previous results showed that the substitution of Cys382 by an alanine,

9 but not that of Cys397, inhibits conidiation and the apical accumulation of both FlbB

10 and FlbE [23].

11 In order to assess the importance of these six cysteines in FlbB and FlbE

12 dynamics and functionality, we followed the mutagenesis procedure described in Figure

13 EV3A to construct a set of mutants where each was substituted with an alanine. All

14 mutant constructs were driven by the $g p d A^{\min i}$ promoter. As can be seen in Figure 3B,

15 only the alanine substitutions of Cys 272 and Cys382 inhibited conidiation and caused a

16 fluffy phenotype as observed previously with the Cys382Ala mutant or the null flbB

17 strain. Moreover, conidia production was decreased in only these two strains (2.5-3.2 x

$1810^{6} \pm 0.9-1.3 \times 10^{6}$ conidia/ $\mathrm{cm}^{2}$ in Cys272Ala, Cys382Ala and null flbB strains; $\mathrm{n}=3$

19 for each strain; $\mathrm{p}=0.00034,0.00055$ and 0.00056 , respectively, in the comparison of

20 fluffy strains with the reference wild-type strain) compared to the wild-type level of

21 production observed in strains expressing wild-type, Cys236Ala, Cys280Ala,

22 Cys303Ala and Cys397Ala GFP::FlbB chimeras $\left(1.5-1.9 \times 10^{7} \pm 0.2-0.6 \times 10^{7}\right.$

23 conidia $/ \mathrm{cm}^{2}$ ) (Figure 3B). All chimeras could be detected by Western-blot and showed

24 the same electrophoretic mobility (Figure EV3B; strains integrating one or two copies

25 of the mutant plasmids were analyzed). The aconidial phenotype of those Cys-to-Ala 
1 mutants correlated with the absence of FlbB and FlbE from the tip (Figure 3C and 3D).

2 Immunoprecipitation experiments between bacterially expressed wild-type or Cys-to-

3 Ala mutant GST::FlbB forms (Cys272Ala; Cys382Ala or the double mutant

4 Cys272Ala;Cys382Ala) and crude protein extracts of a strain expressing FlbE::GFP

5 (Figure 3E) suggested that these two Cys residues are not essential for the interaction

6 between these two UDA-s. Thus, the de-localization of FlbB/FlbE from the tip observed

7 in those mutants was due to other reasons. These results and those showed in previous

8 sections suggest that there is an inter-dependence between FlbB and FlbE for their

9 transport to and accumulation at the tip, and that the incorporation of the complex to the

10 corresponding transport pathway is enabled by specific residues/domains of both

11 proteins.

13 Apical localization of FlbB is altered in a $\triangle m y o E$ background.

14 In cells treated with latrunculin $\mathrm{B}$, which prevents actin monomers from

15 polymerizing, a $g p d A^{m i n i}$-driven GFP::FlbB chimera accumulated in the hyphal subapex

16 but was excluded from the apex ([23]; see also Figure 4A). This meant that, under those

17 conditions, FlbB could move in an acropetal direction and reach the subapex but failed

18 to progress to the apex. Those results suggested that the final stage of the acropetal

19 transport of FlbB was dependent on actin filaments while the transport to the subapex

20 was not. A. nidulans myosin $\mathrm{V}$, MyoE, has been proposed to fuel the actin filament-

21 dependent step of exocytosis [40,41]. Thus, we generated a $\Delta m y o E$ mutant that

22 expressed an $f l b B^{p}$-driven GFP::FlbB chimera and observed that, compared to the wild-

23 type background, FlbB could not gather at the apex and spread into an apical crescent 
1 (Figure 4B). These results suggest that the transport of FlbB from the subapex to the apex occurs through actin filaments and depends on the motor MyoE (see Discussion).

$4 \quad$ Nuclear accumulation of FlbB is inhibited in a nudA1 background.

5 In order to obtain additional information about the dynamics of FlbB at the

6 hyphal tip, we took advantage of the higher apical accumulation of FlbB in the dual-OE

7 strain expressing GFP::FlbB and FlbE::RFP both under the control of $g p d A^{\text {mini }}$ (see the

8 images and kymographs in Figure 4C; and videos EV4A-C). Being that the apical

9 concentration of the bZIP was significantly higher in that strain compared to a

$10 \quad g p d A^{m i n i}:$ :GFP::FlbB strain (see Figure 1A), we expected that this would enable us to

11 track the movements of FlbB more clearly.

13 Figure 4C, left; see also arrowheads in the three kymographs shown and videos EV4B

14 and EV4C). A motionless GFP::FlbB spot could be clearly detected at the subapex in

15 Figure 4C, left (number 2), from which the basipetally moving patches departed (Video

16 EV4A). As the fluorescence intensity decreased as a result of the long exposure times,

17 filament-like fluorescent structures could be observed between the subapex and the

18 apex, which could correspond to GFP::FlbB-decorated actin filaments (number 3 in

19 Figure 4C, left; Video EV4A).

Since the FlbB patches moving towards nuclei seemed to depart from the subapical region corresponding to the dynein loading zone [33], we decided to analyze the nuclear localization of a GFP::FlbB chimera (driven by the native promoter) in a

23 strain expressing the NudA1 thermo-sensitive form of NudA, the heavy chain of dynein 
1 [42]. When wild-type and nudAl backgrounds were compared at $28{ }^{\circ} \mathrm{C}$ (functional

2 NudA1), there was an slight increase in the ratio between the fluorescence intensity at

3 the tip and the most apical nucleus, from $1.37 \pm 0.39$ in the reference GFP::FlbB strain

4 to $1.65 \pm 0.25$ in the $\operatorname{nudAl}$ background ( $\mathrm{n}=12$ and 14 hyphae, respectively; $\mathrm{p}=0.04$;

5 Figure 4D, left). At the restrictive temperature of $37^{\circ} \mathrm{C}$ [43], fluorescent nuclei were

6 hardly detected in the nudAl background (red square in Figure 4D). The fluorescence

7 intensity ratio between the tip and the most apical nucleus significantly increased from

$8 \quad 1.18 \pm 0.42$ in the wild-type to $3.12 \pm 1.79$ in the $n u d A l$ background $(\mathrm{n}=16$ and 27 ,

9 respectively; $\mathrm{p}=0.00013$; since GFP::FlbB was not excluded from nuclei in the mutant

10 we considered the intensity of cytoplasmic fluorescence in hyphae where nuclei could

11 not be discerned). These results support a model in which basipetal transport of FlbB

12 relies principally on the motor complex dynein and its movement towards the minus

13 ends of MTs.

15 FlbD is essential for the nuclear accumulation of FlbB.

FlbB has a close functional relationship with the cMyb-type UDA TF FlbD [30].

17 Both participate in the control of conidiation through cooperative binding to the

18 promoter of $\operatorname{brlA}\left(\operatorname{brl} A^{p}\right)$. Furthermore, chromatin immunoprecipitation assays showed

19 that FlbB cannot bind $\operatorname{brlA}^{p}$ in the absence of FlbD [30]. These preliminary results

20 suggest that FlbD plays an important role in the transcriptional activity of FlbB, but it is

21 unknown if the cMyb factor is required for the nuclear accumulation of the bZIP.

22 Consequently, we analyzed the localization of FlbB::GFP, driven by the native

23 promoter, in a $\Delta f l b D$ strain that co-expressed the histone $\mathrm{H} 1$, HhoA, fused to mCherry

24 [44], as a marker for the nuclei (Figure 5A, left). A statistically significant inhibition of 
1 the nuclear localization of FlbB::GFP was observed in the null $f l b D$ strain, together with

2 increased GFP fluorescence in the apex. The fluorescence intensity ratio between the tip

3 and the most apical nucleus increased from $1.50 \pm 0.40$ in the wild-type to $13.90 \pm 3.00$

4 in the null $f l b D$ strain $\left(\mathrm{n}=10\right.$ hyphae for each strain; $\mathrm{p}=4.41 \times 10^{-12}$; see the graphs in

5 Figure 5A).

Visualizing FlbB movement in vegetative hyphae is difficult. It cannot be

7 detected when FlbB::GFP expression is driven by the native promoter, it can be barely

8 detected near the tip when GFP::FlbB expression is driven by the $g p d A^{\min i}$ promoter

9 [23], and can only be followed when both FlbB and FlbE are expressed constitutively

10 (see above in Figure 4C). Interestingly, deletion of $f l b D$ allowed the observation of

11 FlbB::GFP patches $\left(f l b B^{p}\right.$ promoter) moving bidirectionally along the cytoplasm (red

12 and black arrows, respectively; Figure 5A, middle). To check if the bidirectional long-

13 distance cytoplasmic movement of FlbB::GFP in the $\Delta f l b D$ background was MT-

14 dependent, we analyzed FlbB dynamics in a medium containing $3 \mu \mathrm{g} / \mathrm{ml}$ benomyl [45].

15 The addition of the drug clearly inhibited FlbB::GFP movement, as shown by the

16 vertical lines observed in the kymograph in Figure 5A, right. These results show that in

17 the absence of FlbD, FlbB is not accumulated in nuclei and suggest that it remains

18 moving in both directions along the cytoplasm in a MT-dependent manner.

19 The above results also suggest that the quantity of FlbB that can be accumulated

20 in nuclei is directly related to FlbD levels. If this hypothesis holds true, overexpression

21 of $f l b D$ should correlate with a higher nuclear accumulation of the bZIP factor. Thus,

$22 f l b D$ overexpression was induced through the alcA promoter, $a l c A^{p}$ [30]. According to

23 Wieser and coworkers, over-expression of $f l b D$ triggers the development of

24 conidiophores in shaken cultures, after the transference of mycelia from a liquid

25 medium supplemented with glucose as the carbon source (represses $a l c A^{p}$ ) to a medium 
1 with threonine (alcA $A^{p}$ induction) [46]. This was confirmed for an $a l c A^{p}:: f l b D$ strain

2 expressing FlbB::GFP (Figure EV5A).

EtOH $(1 \%)$ and threonine $(100 \mathrm{mM})$ were assessed as possible carbon sources

4 inducing $a l c A^{p}$-mediated $f l b D$ overexpression, on solid ACM medium and in

5 comparison with the phenotypes of the reference FlbB::GFP and $\Delta f l b D$ strains (Figure

6 EV5B). In general, all strains produced more aerial hyphae when EtOH was used as the

7 carbon source. The use of threonine, however, induced clear phenotypic differences

8 between $a l c A^{p}:: f l b D\left(1.0 \times 10^{7} \pm 2.2 \times 10^{6}\right.$ conidia/ $\left.\mathrm{cm}^{2}\right)$ or $a l c A^{p}:: f l b D ;$ FlbB::GFP $(1.2$

$9 \times 10^{7} \pm 1.2 \times 10^{6}$ conidia/ $\left./ \mathrm{cm}^{2}\right)$ and the reference FlbB::GFP strain $\left(5.5 \times 10^{6} \pm 1.7 \times 10^{6}\right.$

10 condia $/ \mathrm{cm}^{2} ; \mathrm{n}=3$ for each strain; $\mathrm{p}=0.05$ and 0.005 , respectively).

11 Considering the results described above, glucose (repressor) or threonine

12 (inducer) were used in the analysis of the subcellular localization of FlbB::GFP in wild-

13 type or alc $A^{p}:: f l b D$ genetic backgrounds (Figure EV5C). Under repressing conditions,

14 FlbB::GFP (wild-type background) localized, as expected, to the tip and the most apical

15 nucleus. The calculated fluorescence intensity ratio between the tip and the most apical

16 nucleus was $1.42 \pm 0.39$ in this case $(n=10)$. This ratio increased in the same medium

17 to $4.52 \pm 2.05$ in the $a l c A^{p}:: f l b D$ background $(\mathrm{n}=10 ; \mathrm{p}=0.00012)$, a significant change

18 that was caused by the decrease in the nuclear localization of FlbB observed when $f l b D$

19 expression was repressed (as before, cytoplasmic fluorescence was considered as the

20 value of nuclear fluorescence) (Figure EV5C, upper-right panel). This localization

21 resembled qualitatively what was observed in a $\Delta f l b D$ background (Figure EV5C,

22 bottom-left). Under conditions inducing $a l c A^{p}$, FlbB::GFP recovered the nuclear

23 localization (Figure EV5C, bottom-right), decreasing the fluorescence intensity ratio

24 between the tip and the most apical nucleus to $1.15 \pm 0.14(\mathrm{n}=15 ; \mathrm{p}=0.000001$

25 compared to the same strain under repressing conditions). Taken together, these results 
1 suggest that the $a l c A^{p}$-mediated upregulation of $f l b D$ in threonine-containing medium

2 increases the nuclear localization of FlbB. Nevertheless, this observation cannot be

3 directly linked to the induction of conidiophore development described in Figure EV5A

4 (shaken cultures) because these fluorescence microscopy analyses were carried out with

5 static instead of shaken cultures. Taken together, the results shown in this section

$6 \quad$ strongly suggest that FlbD is a key element for the nuclear accumulation of FlbB.

8 C- and N-termini of FlbD are necessary for conidiation.

The role of FlbD in conidiation and the nuclear accumulation of FlbB was then

10 analyzed in more detail. First, we observed that strains expressing N- or C-terminal

$11 \mathrm{HA}_{3 \mathrm{x}}$-tagged versions of FlbD showed a delay in conidiation compared to that

12 expressing an FlbD::GFP chimera (Figure EV5D). Conidia production in $\mathrm{HA}_{3 \mathrm{x}}$-tagged

13 strains was significantly lower than in reference wild-type or FlbD::GFP strains after 48

14 hours of culture in AMM plates $\left(4.0-4.2 \times 10^{7} \pm 0.6-1.0 \times 10^{7}\right.$ conidia $/ \mathrm{cm}^{2}$ in reference

15 strains; $1.0-1.7 \times 10^{7} \pm 0.2-0.3 \times 10^{7}$ conidia $/ \mathrm{cm}^{2}$ in $\mathrm{HA}_{3 \mathrm{x}}$-tagged strains; $\mathrm{p}=0.65$ when

16 the strain expressing a FlbD::GFP chimera and the wild-type strain were compared; $\mathrm{p}=$

$170.0000051,0.00000090$ or 0.00000024 when strains expressing FlbD::HA $\mathrm{HA}_{3 \mathrm{x}}$,

$18 \mathrm{HA}_{3 \mathrm{x}}:$ :FlbD or $\mathrm{HA}_{3 \mathrm{x}}::$ FlbD::GFP chimeras were compared to the reference strain; $\mathrm{n}=3$

19 replicates for each strain) (Figure EV5D). These results suggest that $\mathrm{HA}_{3 \mathrm{x}}$ (but not GFP)

20 tagging of FlbD partially hinders its activity.

21 In an attempt to explain this apparent contradiction $\left(\mathrm{HA}_{3 \mathrm{x}}\right.$ tag contains nine

22 times less amino acids than GFP), the sequence of FlbD was analyzed. FlbD orthologs

23 were found in Eurotiomycetes (Eurotiales, Onygenales) and Sordariomycetes classes,

24 being the orthologs of this last class the most divergent ones [47]. An alignment of 
1 orthologs of Eurotiales differentiated five conserved domains but only two of them, the

2 cMyb transcriptional regulatory domain, which is located at the N-terminus, and a small

3 domain at the C-terminus (residues 303-314), were conserved in all orthologs (Figure

$4 \quad 5 \mathrm{~B}$ and Appendix Figure S1). Thus, we hypothesized that tagging at either the N- or C-

5 termini could partially inhibit FlbD function, delaying conidiation. However, the fact

6 that $\mathrm{HA}_{3 \mathrm{x}}$-tagging but not GFP-tagging delayed conidiation was unexpected.

Since the strain expressing FlbD::GFP conidiated as the wild-type, it could be

8 suggested that the transcript or protein chimera was unstable and therefore truncated,

9 giving an active but untagged version of the protein. A strain expressing an

$10 \mathrm{HA}_{3 \mathrm{x}}:$ :FlbD::GFP chimera was, thus, generated to confirm this hypothesis through

11 immunodetection experiments (Figure EV5E). Two bands were detected when protein

12 extracts of this strain were hybridized with an $\alpha-\mathrm{HA}_{3 \mathrm{x}}$ antibody, one corresponding to

13 the whole chimera and the second one at a size slightly bigger than that of $\mathrm{HA}_{3 \mathrm{x}}: \mathrm{FlbD}$

14 (probably including some amino acids of the N-terminus of GFP). Taken together, these

15 results explain the low fluorescence intensity levels shown by FlbD::GFP [30] and are

16 consistent with both the N-terminal transcriptional regulatory domain and the C-

17 terminal domain playing an important role in FlbD activity.

18 The study of FlbD forms bearing specific substitutions within the N-terminal

19 region has shown that the $\mathrm{cMyb}$ transcriptional regulatory domain is essential to induce

20 or complete both asexual and sexual cycles [48], but there is no information on this

21 region's hypothetical role in the nuclear accumulation of FlbB. Additionally, the

22 Eukaryotic linear motif (ELM) resource for functional site prediction in proteins

23 (http://elm.eu.org/) suggested that the last C-terminal amino acids of FlbD could

24 correspond to a LIG_NRBOX motif (amino acids 308-314; expect value: $2.63 \mathrm{e}^{-04}$ ),

25 which supposedly confers the ability to bind nuclear receptors and is found primarily in 
1 co-activators of those receptors (http://elm.eu.org/elms/LIG_NRBOX.html).

2 Considering the short length of this domain (LxxLL) and the low expect value reported,

3 its presence in FlbD could be meaningless. Thus, we identified all A. nidulans proteins

4 predictably containing a LIG_NRBOX domain (2,227 proteins) and observed that

5 transcription factors were significantly enriched in that motif compared to proteins

6 associated to other gene ontology terms (see Appendix Table S1). Therefore, we judged

7 that informatic support of the LxxLL motif of FlbD being functional justified further

8 investigation.

9 Using a site-directed mutagenesis approach similar to that one followed for flbE

10 mutagenesis (Figure EV1C), strains expressing a mutant FlbD $^{(\mathrm{L} 309 \mathrm{~A} ; \mathrm{L} 312 \mathrm{~A})}$ form or a

11 truncated $\mathrm{FlbD}^{(1-112)}$ form were generated. In addition, using a random mutagenesis

12 approach, an aconidial mutant bearing two point mutations in codons corresponding to

13 the first and second cMyb domains of FlbD (E14G and R87Q) was isolated. The

14 phenotype of these three mutant strains was compared to those of wild-type and null

$15 f l b D$ strains (Figure 5C). After 72 hours of culture in AMM, conidia production

16 decreased significantly in all mutants compared to the wild-type strain $(p=0.0030$,

170.0002 and 0.0152 in strains expressing $\mathrm{FlbD}^{(1-112)}, \mathrm{FlbD}^{(\mathrm{E} 14 \mathrm{G} ; \mathrm{R} 87 \mathrm{Q})}$ or $\mathrm{FlbD}^{(\mathrm{L} 309 \mathrm{~A} ; \mathrm{L} 312 \mathrm{~A})}$

18 forms, respectively; $n=6$ for each strain). All these three mutations caused a significant

19 decrease in the ratio between the apical and nuclear fluorescence intensities of FlbB

20 compared to the reference background $(\mathrm{p}=0.00056,0.0000011$ and 0.0014 ,

21 respectively; $\mathrm{n}>13$ hyphae for each strain) (see Figure 5D), strongly suggesting that

22 besides the DNA-binding domain of FlbD (D1; cMyb), a predicted LIG_NRBOX motif

23 (D5) plays an important role in the nuclear accumulation of the bZIP factor FlbB.

As occurred in FlbD, the presence of a LxxLL sequence was also observed in

25 FlbB (L330 to L334), but not in other TFs known to bind the promoter of $b r l A$, such as 
1 FlbC, VosA, NsdD or AbaA. The last two Leu residues of this domain were replaced by

2 alanines, $\mathrm{FlbB}^{(\mathrm{L} 333 \mathrm{~A} ; \mathrm{L} 334 \mathrm{~A})}$, causing a significant decrease in the production of conidia

3 (Figure 5E), from $5.43 \times 10^{7} \pm 0.2 \times 10^{7}$ in the reference GFP::FlbB strain to $0.71 \times 10^{7}$

$4 \pm 0.3 \times 10^{7}$ conidia $/ \mathrm{cm}^{2}$ in the mutant strain $(\mathrm{p}=0.0000192 ; \mathrm{n}=3$ for each strain $)$. This

5 phenotype correlated with a significant decrease in the nuclear fluorescence of FlbB.

6 The ratio between the fluorescence intensity at the tip and the most apical nucleus

7 increased from $1.49 \pm 0.44$ in the reference strain to $2.26 \pm 0.50$ in the double-leucine

8 mutant of FlbB ( $p=0.000029 ; \mathrm{n}=14$ and 24 hyphae, respectively) (Figure 5F). These

9 results support the above-mentioned hypothesis that LxxLL motifs mediate the nuclear

10 localization of UDA TFs FlbB and FlbD.

12 Discussion.

13 The activation of the production of asexual multicellular structures in 14 Aspergillus nidulans largely (but not exclusively) relies on the signal transduction 15 pathway controlled by FlbB, FlbE and FlbD. A key element for the timely regulation of 16 brlA expression is the spatio-temporal control in vegetative hyphae of the dynamics of 17 FlbB, which has to be first transported to the hyphal tip and from there imported into 18 nuclei [23]. Both FlbE and FlbD show a close functional relationship with FlbB and 19 play key roles in this process, but clearly differentiated in space and time. The 20 interaction with FlbE enables acropetal transport and accumulation of FlbB at the tip

21 while FlbD is essential for the nuclear localization of the pool of transcriptionally active

22 FlbB generated at the growth region. The available information and our hypotheses on

23 the molecular basis of this sequence of events has been summarized in Figure 6 and will

24 be used to structure this discussion. 


\section{Acropetal transport mechanism.}

2

3

4 transcriptionally active pool of FlbB described when only FlbB is expressed

5 constitutively decreases as the quantity of FlbE increases and disappears in those strains

6 expressing FlbE and FlbB fused in the same chimera $\left(g p d A^{\min i}\right.$-driven FlbE::FlbB::GFP

7 or FlbE::mRFP::T2A $\left.{ }^{(\mathrm{G} 17 \mathrm{~A})}:: \mathrm{FlbB}:: \mathrm{GFP}\right)$. These results suggest that the FlbB/FlbE

8 complex is composed of equimolar amounts of each developmental regulator, although

9 the stoichiometry of the complex (i.e., 1:1 or 2:2) is still unknown (Figure 6A, 6B and

10 6C). While the bZIP domain of FlbB is essential and sufficient for this

11 heterodimerization and to date FlbB homodimers have not been detected [23], cysteine

12 residues in positions 272 and 382 apparently play a modulatory role (Figure 6C).

13 However, that these cysteines are essential for the apical accumulation of the complex,

14 strongly suggests that FlbB does not join its acropetal transport pathway exclusively

15 through FlbE and that its cysteines, probably in combination with additional elements,

16 play a key role (see the legend of Figure 6C).

The role of FlbE in the subcellular dynamics of FlbB seems to be limited exclusively to acropetal transport and at least five of its seven domains (E1, E4, E5, the linker domain and to lesser extent E3) [34] are necessary for the apical accumulation of the bZIP TF (Figure 6B and 6C). Considering that FlbE interacts with the bZIP domain

21 of FlbB (but apparently not with central and C-terminal domains) and the presence of a 22 nuclear localization signal (NLS) prior to the bZIP [23], it is tempting to suggest that 23 besides assisting the acropetal transport of the complex, FlbE binding could occlude the $24 \quad$ NLS of FlbB, precluding its basipetal transport and nuclear import. 
The characteristics of FlbB transport towards the polarity site as well as the

2 possibility of domain E1 of FlbE being a signal peptide open the possibility of the

3 incorporation of the FlbB/FlbE complex into a vesicular fraction that would transit

4 through the ER-Golgi network (Figure 6B). In preliminary LC-MS/MS-coupled co-

5 immunoprecipitation assays of protein extracts of a strain expressing the $g p d A^{\operatorname{mini}}$-driven

6 GFP::FlbB chimera, we identified several proteins participating in the transport between

7 the ER and the Golgi apparatus. These preliminary results correlate with the hypothesis

8 proposed above and at the same time open an avenue for a future, comprehensive

9 analysis of how these two developmental regulators join the secretory pathway, which

10 additional proteins they establish interactions with, which of their domains are required

11 or what could be the conformation and stoichiometry of the complex. The transit of

12 vesicles between the ER and the Golgi apparatus is MT dependent, while FlbB reaches

13 the apex of hyphae in a culture medium containing benomyl, which destabilizes MTs

14 [23]. Thus, additional experiments are required to elucidate the hypothetic mechanism

15 of FlbB/FlbE transition through the ER-Golgi network.

In the absence of actin polymerization, FlbB reaches the subapex but fails to

17 accumulate in the apex [23]. In a null myoE background, FlbB spreads into an apical

18 crescent that resembles the localization shown in that genetic background by the post

19 Golgi-carrier marker RabE/Rab11 [41,43]. As Pantazopoulou and collaborators found in

20 their characterization of RabE, it could be suggested that, without MyoE activity, FlbB

21 might be captured by the "actin mop" but lacked a molecular motor which could deliver

22 it to the apex [41]. Thus, myosin V (MyoE) arises as the best candidate motor protein to

23 deliver FlbB, on actin filaments, from the subapex to the apex (Figure 6B).

\section{Basipetal transport and nuclear accumulation of FlbB.}


2 in its journey towards nuclei (Figure 6B). In accordance with the long-distance basipetal

3 transport of vesicles and macromolecular cargo in neurons, it is generally accepted in $A$.

$4 \quad$ nidulans hyphae that vesicles formed by endocytosis at the subapex are bound by the

5 dynein complex and transported on MTs towards distal regions [49]. The inhibition of

6 the nuclear accumulation of FlbB observed in a thermo-sensitive heavy-chain dynein

7 nudAl background at the restrictive temperature $\left(37^{\circ} \mathrm{C}\right)$ correlates with a model in

8 which FlbB follows this pathway. This, at the same time, is in agreement with the MT-

9 dependence of the cytoplasmic, bidirectional transport of FlbB observed in a $\Delta f l b D$

10 background, which suggests that in the absence of FlbD, a fraction of FlbB remains

11 moving bidirectionally along the length of the cytoplasm. An interesting question for

12 the future will be the elucidation of how FlbB joins the dynein-mediated basipetal

13 transport pathway and the identification of adaptor proteins and the karyopherins

14 required for its nuclear accumulation. This will open the possibility of a deeper analysis

15 of the role of the NLS and the LxxLL motif of FlbB, both of them required for the

16 nuclear accumulation of the TF (Figure 6B and 6D).

In the absence of FlbD, FlbB does not accumulate in nuclei and cannot bind $\operatorname{brl}^{p}$ [30], thus inhibiting conidiation. Besides offering the possibility of using the null

$19 f l b D$ strain to identify proteins required for the basipetal transport and nuclear

20 accumulation of FlbB, these results raise the following question: which is the primary

21 cause of the inability to trigger asexual development in this background. It is clear that

22 the absence of FlbB from nuclei impedes binding to $b r l A^{p}$ but, at the same time, FlbB

23 accumulation in nuclei may be reduced due to an inability to bind DNA in the absence

24 of FlbD activity (Figure 6B and 6E). The only subcellular localization described for

25 FlbD and the orthologs that have been functionally characterized is nuclear [30,50,51], 
1 suggesting that it is not directly involved in the basipetal transport of FlbB but in its

2 nuclear retention. Our results show that both the $\mathrm{N}$ - and $\mathrm{C}$-termini of FlbD are necessary

3 for the induction of conidiation and the nuclear accumulation of FlbB. Since its N-

4 terminal cMyb transcriptional regulatory domain is sufficient for FlbD to bind $b r l A^{p}$

5 [30], a link between DNA-binding by FlbD and the nuclear accumulation of FlbB (and

6 perhaps DNA-binding by the bZIP TF) can be suggested. In this context, the possibility

7 of FlbD acting as a pioneer TF is open [52], binding $b_{r l}^{p}$ first [30], causing a

8 modification of the conformation of chromatin and enabling then binding of FlbB

9 (Figure 6E) [52,53]. Alternatively (or in addition), the cMyb domain of FlbD could act

10 as a heterodimerization domain for the bZIP of FlbB [53], forming a heterocomplex

11 which, in turn, is capable of binding to the targets of each TF within $b r l A^{p}$, which are

12 predicted to be adjacent (Figure 6E) [30]. In both scenarios, LxxLL motifs of both FlbD

13 and FlbB may play a modulatory role and/or mediate in the interaction with additional

14 elements. Although both TFs bind to a common region of 300 nt-s within $b r l A^{p}$, future

15 experiments must determine the nature of the exact target-DNA sequences of both FlbB

16 and FlbD in $b r l A^{p}$, the hierarchy/democracy between both TFs and the study of

17 hypothetical modifications in the structure of chromatin at this region $[54,55]$. The

18 extension of these analyses to other activators and repressors that bind $b r l A^{p}$ (Lee et al.,

19 2016) will further a deeper understanding of how TFs belonging to different pathways

20 are coordinated for a timely control of multicellular development.

22 Materials and Methods.

23 Oligonucleotides, strains and culture conditions. 
Appendix Tables S3 and S4 show, respectively, the oligonucleotides and the strains of A. nidulans used in this work. Strains were cultivated in supplemented liquid or solid Aspergillus minimal (AMM) or complete (ACM) media [56,57], using glucose

$4(2 \%)$ and ammonium tartrate $(5 \mathrm{mM})$ as carbon and nitrogen sources, respectively.

5 Fermentation medium (AFM), which contained 25g/L corn steep liquor (Sigma-

6 Aldrich) and sucrose $(0.09 \mathrm{M})$ as the carbon source, was used to culture samples for

7 protein extraction [58]. Mycelia for DNA extraction and Southern-blot analysis were

8 cultured in liquid AMM and the procedures described previously by us were followed 9 [34].

10 Conidia production on solid medium was calculated as described previously by 11 us [30], using three replicates for each strain. The two-tailed Student's t-test for

12 unpaired samples was used to determine the statistical significance of the changes in 13 conidia production.

Gene overexpression through the use of $a l c A^{\mathrm{p}}$ was induced in solid medium that

15 contained threonine $(100 \mathrm{mM})$ and repressed when glucose $(2 \%)$ was used. For the 16 alc $A^{p}$-mediated overexpression in liquid culture, first $10^{6}$ conidia per milliliter were 17 cultured at $37{ }^{\circ} \mathrm{C}$ for 18 hours in standard AMM. Then, mycelia samples were filtered 18 and transferred to AMM that contained threonine $(100 \mathrm{mM})$ as the carbon source, with 19 additional 20 hours of culture [59]. Hyphal and conidiophore morphology were then 20 analyzed using a Nikon Optiphot microscope, coupled to a Nikon FX-35DX camera.

22 of A. nidulans strains in supplemented watch minimal medium (WMM) [60] and 23 incubating them for 18 hours at room temperature. 
As a sample and a tutorial of the multiple advantages for genetic manipulation

3 wild-type or mutant forms of the proteins of interest, expressed through native or

4 constitutive promoters, and tagged at $\mathrm{N}$ - or C-termini are briefly described along the

5 results section as expanded view figures. Most of those procedures, as well as those

6 followed for the generation of deletants, are based on the fusion-PCR technique

7 developed by Yang and colleagues and the subsequent protoplast transformation

8 protocol developed by Tilburn and colleagues or Szewczyk and colleagues [61-63].

9 Cys-to-Ala mutants of FlbB were generated by transforming protoplasts with mutant

$10 \operatorname{pgpdA}^{\min i}:: \mathrm{GFP}:: \mathrm{FlbB}^{\text {(Cys-to-Ala) }}$ plasmids. Recombination of these mutant plasmids was

11 directed to the pyroA locus. The strain expressing GFP::FlbB in a nudAl genetic

12 background was generated by meiotic crosses [64].

\section{Fluorescence microscopy.}

Subcelllar localization of FlbB and FlbE in hyphae was analyzed as previously

15 described using a Leica DMI-6000b or a Zeiss Axio Observer Z1 inverted microscopes

$16[23,65]$. The former is equipped with a 63x Plan Apo 1.4 N.A. oil immersion lens from

17 Leica, and filters GFP (excitation at $470 \mathrm{~nm}$ and emission at $525 \mathrm{~nm}$ ) and Txred

18 (excitation at $562 \mathrm{~nm}$ and emission at $624 \mathrm{~nm}$ ). The latter includes a $63 \mathrm{x}$ Plan

19 Apochromat 1.4 oil immersion lens, and filters 38 (excitation at $470 \mathrm{~nm}$ and emission at

$20525 \mathrm{~nm}$ ) and 43 (excitation at $545 \mathrm{~nm}$ and emission at $605 \mathrm{~nm}$ ). Fluorescence levels

21 were measured using ImageJ software (http://imagej.nih.gov/ij) (U. S. National

22 Institutes of Health, Bethesda, Maryland, USA).

\section{Protein extraction and immunodetection.}


Two different protocols were used for protein extraction. Direct

2 immunodetection of proteins was done using protein extracts that were obtained through

3 the alkaline lysis protocol [66], which prevents protein degradation. Briefly,

4 approximately $6 \mathrm{mg}$ of lyophilized mycelium were resuspended in $1 \mathrm{ml}$ lysis buffer

$5(0.2 \mathrm{M} \mathrm{NaOH}, 0.2 \% \beta$-mercaptoethanol). After trichloroacetid acid (TCA) precipitation,

$6100 \mu \mathrm{l}$ Tris-Base $(1 \mathrm{M})$ and $200 \mu \mathrm{l}$ of loading buffer $(62.5 \mathrm{mM}$ Tris- $\mathrm{HCl} \mathrm{pH}=6.8,2 \%$

$7 \operatorname{SDS}(\mathrm{p} / \mathrm{v}), 5 \%$-mercaptoethanol (v/v), $6 \mathrm{M}$ urea and $0.05 \%$ bromophenol blue $(\mathrm{p} / \mathrm{v}))$

8 were added. Samples were then loaded on polyacrilamide gels $(\% 10)$ for protein-content

9 assessment and Western-blot analysis.

10 For co-immunoprecipitation assays, protein extracts were obtained through the

11 procedure described by [67]. Samples were lyophilized and pulverized before the

12 addition of $1 \mathrm{ml}$ of NP-40 extraction buffer (5 mM Hepes pH=7.5, $1 \mathrm{mM}$ EDTA, 20

$13 \mathrm{mM} \mathrm{KCl}, 0.1 \% \mathrm{NP}-40,0.5 \mathrm{mM}$ DTT and a protease inhibitor cocktail from Roche; plus

$14150 \mathrm{mM} \mathrm{NaCl}$ when the extract was going to be incubated with Chromotek's GFP-Trap

15 beads). The Bradford assay was used for the determination of protein concentration.

16 Before the use of crude extracts in co-immunoprecipitation assays, $200 \mu \mathrm{g}$ of protein

17 were precipitated with TCA and purified with ethanol/eter mixes (1:1 and 1:3,

18 respectively). After resuspension in $80 \mu \mathrm{l}$ of loading-buffer, the integrity of samples was

19 assessed by polyacrilamide gel electrophoresis while expression of the chimera of

20 interest was confirmed by Western-blot.

For immunodetection analyses, proteins were separated in standard 10\% SDS-

22 polyacrylamide gels before electro-transferring them to nitrocellulose filters. GFP-,

23 mRFP- or $\mathrm{HA}_{3 x}$-tagged proteins were detected with $\alpha$-GFP (mouse), $\alpha$-RFP (rabbit) or

$24 \alpha-\mathrm{HA}_{3 \mathrm{x}}$ (mouse) monoclonal antibody cocktails (1:5000 Roche, 1:4000 USBiological 
1 and 1:1000 Santa Cruz, respectively). Peroxidase conjugated $\alpha$-mouse or $\alpha$-rabbit

2 (1/4000, Jackson ImmunoResearch Laboratories, or 1:10000, Sigma Aldrich,

3 respectively) were used as secondary antibody. Peroxidase activity was induced with

4 Amersham Biosciences ECL kit, and chemiluminescence was detected using a

5 Chemidoc $+\square$ XRS system (Bio-Rad).

\section{Expression of recombinant proteins in Bacteria.}

Plasmid pGEX-2T (Pharmacia) was used to express fusions of GST to full (1-

8 426) or point-mutant (C272A, C382A and C272A;C382A) versions of FlbB. After

9 culturing transformant E. coli DH1 cells until OD600 $\square=\square 0.6-0.8$, expression of GST-

10 tagged proteins was induced with the addition of $0.1 \square \mathrm{mM}$ IPTG and further incubation

11 at $15^{\circ} \mathrm{C}$ for $24 \square \mathrm{h}$. Extracts containing recombinant GST chimeras were then obtained

12 essentially as described by us previously [23].

13 Co-immunoprecipitation assays.

14 Two procedures were followed. The first one analyzed the ability of the above-

15 mentioned GST-tagged FlbB chimeras, used as baits, to retain wild-type and mutant

16 forms of FlbE::GFP [23]. Briefly, GST-tagged proteins attached to glutathione

17 sepharose media (GE Healthcare) were incubated at $4^{\circ} \mathrm{C}$ for $1 \square \mathrm{h}$ with $2-3 \mathrm{mg}$ of crude

18 protein extracts of $A$. nidulans. After 3-5 washing steps (a sample of this non-retained,

19 NR, fraction was stored for analysis), loading buffer was used to resuspended the resin

20 (retained fraction, R). Proteins were visualized with the Bio-Safe Coomassie stain (Bio-

21 Rad), and tagged proteins were specifically detected by immunodetection. 
1 Hepes $\mathrm{pH}=7.5,150 \mathrm{mM} \mathrm{NaCl}$ and $0.5 \mathrm{mM}$ EDTA) and incubated with $6 \mathrm{mg}$ of the

2 protein extract for 90 minutes at $4{ }^{\circ} \mathrm{C}$. After centrifugation, the supernatant was

3 precipitated in TCA, resuspended in loading buffer and stored as the non-retained (NR)

4 fraction. The resin was then washed three times using protein extraction buffer plus 150

$5 \mathrm{mM} \mathrm{NaCl}$ (see before) and finally resuspended in loading buffer (R: retained fraction).

$6 \quad$ Both NR and R fractions were resolved by SDS-PAGE electrophoresis.

\section{Author contribution.}

9 O.E., E.A.E., A.O., E.P-A. and E.O-A designed and generated the strains, and carried

10 out the experiments. O.E. and E.A.E. supervised the experimental part. O.E. wrote the

11 manuscript. M.S.C. performed bioinformatic analyses. All authors contributed to the

12 improvement of the text and figures.

14 Funding information.

15 Work at the UPV/EHU lab was funded by UPV/EHU (grant EHUA15/08 to

16 O.E) and the Basque Government (grant IT599-13 to Dr. Unai Ugalde). Work at CIB-

17 CSIC was funded by MINECO (BFU2015-66806-R to E.A.E). E.P-A and E.O-A held

18 predoctoral fellowships from UPV/EHU. A.O held a predoctoral fellowship from the

19 Basque Government.

\section{Acknowledgements.}


3 flbE mutants (W11A) and the strains expressing the T2A-tagged forms, as well as the

4 bioinformatic analysis of FlbD.

6 Conflict of interest.

No conflict of interest declared. 
1

3

4

6

7

8

9

10

11

12

13

14

15

16

17

18

19

20

21

22

23

24

25

26

27

28

29

\section{References.}

1. Bradshaw RA, Dennis EA (2010) Cell Signaling: Yesterday, Today, and Tomorrow. In Bradshaw RA, Dennis EABT-H of CS (Second E (eds.) pp 1-4. Academic Press, San Diego.

2. Rishal I, Kam N, Perry RB-T, Shinder V, Fisher EMC, Schiavo G, Fainzilber M (2012) A motor-driven mechanism for cell-length sensing. Cell Rep 1: 608-616.

3. Bielska E, Higuchi Y, Schuster M, Steinberg N, Kilaru S, Talbot NJ, Steinberg G (2014) Long-distance endosome trafficking drives fungal effector production during plant infection. Nat Commun 6: 5097.

4. Ramos-García SL, Roberson RW, Freitag M, Bartnicki-García S, Mouriño-Pérez RR (2009) Cytoplasmic Bulk Flow Propels Nuclei in Mature Hyphae of Neurospora crassa. Eukaryot Cell 8: 1880 LP-1890.

5. Etxebeste O, Espeso EA (2016) Neurons show the path: Tip-to-nucleus communication in filamentous fungal development and pathogenesisa. FEMS Microbiol Rev 40:.

6. Panayotis N, Karpova A, Kreutz MR, Fainzilber M (2015) Macromolecular transport in synapse to nucleus communication. Trends Neurosci 38: 108-116.

7. Terenzio M, Koley S, Samra N, Rishal I, Zhao Q, Sahoo PK, Urisman A, Marvaldi L, Oses-Prieto JA, Forester C, et al. (2018) Locally translated mTOR controls axonal local translation in nerve injury. Science (80- ) 359: 1416 LP1421.

8. Ben-Yaakov K, Dagan SY, Segal-Ruder Y, Shalem O, Vuppalanchi D, Willis DE, Yudin D, Rishal I, Rother F, Bader M, et al. (2012) Axonal transcription factors signal retrogradely in lesioned peripheral nerve. EMBO J 31: 1350-1363.

9. Takeshita N, Evangelinos M, Zhou L, Serizawa T, Somera-Fajardo RA, Lu L, Takaya N, Nienhaus GU, Fischer R (2017) Pulses of Ca2+ coordinate actin assembly and exocytosis for stepwise cell extension. Proc Natl Acad Sci 114: 5701-5706.

10. Riquelme $M$ (2013) Tip growth in filamentous fungi: a road trip to the apex. Annu Rev Microbiol 67: 587-609.

11. Riquelme M, Aguirre J, Bartnicki-García S, Braus GH, Feldbrügge M, Fleig U, Hansberg W, Herrera-Estrella A, Kämper J, Kück U, et al. (2018) Fungal Morphogenesis, from the Polarized Growth of Hyphae to Complex Reproduction and Infection Structures. Microbiol Mol Biol Rev 82:.

12. Turrà D, Di Pietro A (2015) Chemotropic sensing in fungus-plant interactions. Curr Opin Plant Biol 26: 135-140.

13. Turrà D, El Ghalid M, Rossi F, Di Pietro A (2015) Fungal pathogen uses sex pheromone receptor for chemotropic sensing of host plant signals. Nature 527: 521. 
14. Masachis S, Segorbe D, Turrà D, Leon-Ruiz M, Fürst U, El Ghalid M, Leonard G, López-Berges MS, Richards TA, Felix G, et al. (2016) A fungal pathogen secretes plant alkalinizing peptides to increase infection. Nat Microbiol 1: 16043.

15. Stephenson KS, Gow NAR, Davidson FA, Gadd GM (2014) Regulation of vectorial supply of vesicles to the hyphal tip determines thigmotropism in Neurospora crassa. Fungal Biol 118: 287-294.

16. Brand AC, Morrison E, Milne S, Gonia S, Gale CA, Gow NAR (2014) Cdc42 GTPase dynamics control directional growth responses. Proc Natl Acad Sci 111: 811 LP-816.

17. Brand A, Gow NAR (2012) Tropic Orientation Responses of Pathogenic Fungi BT - Morphogenesis and Pathogenicity in Fungi. In Pérez-Martín J, Di Pietro A (eds.) pp 21-41. Springer Berlin Heidelberg, Berlin, Heidelberg.

18. Mooney JL, Yager LN (1990) Light is required for conidiation in Aspergillus nidulans. Genes Dev 4: 1473-1482.

19. Skromne I, Sanchez O, Aguirre J (1995) Starvation stress modulates the expression of the Aspergillus nidulans brlA regulatory gene. Microbiology 141: 21-28.

20. Adams $\mathrm{TH}$, Wieser JK, Yu J-H (1998) Asexual sporulation in Aspergillus nidulans. Microbiol Mol Biol Rev 62: 35-54.

21. Rodríguez-Urra AB, Jiménez C, Nieto MI, Rodríguez J, Hayashi H, Ugalde U (2012) Signaling the Induction of Sporulation Involves the Interaction of Two Secondary Metabolites in Aspergillus nidulans. ACS Chem Biol 7: 599-606.

22. Ugalde U, Rodriguez-Urra AB (2014) The Mycelium Blueprint: insights into the cues that shape the filamentous fungal colony. Appl Microbiol Biotechnol 98: 8809-8819.

23. Herrero-Garcia E, Perez-de-Nanclares-Arregi E, Cortese MS, MarkinaIñarrairaegui A, Oiartzabal-Arano E, Etxebeste O, Ugalde U, Espeso EA (2015) Tip-to-nucleus migration dynamics of the asexual development regulator FlbB in vegetative cells. Mol Microbiol 98: 607-624.

24. Etxebeste O, Villarino M, Markina-Iñarrairaegui A, Araújo-Bazán L, Espeso EA, Thompson B, Cheng P, Poo M, Hatanaka Y, Yamauchi K, et al. (2013) Cytoplasmic dynamics of the general nuclear import machinery in apically growing syncytial cells. PLoS One 8: e85076.

25. Bayram Ö, Bayram ÖS, Ahmed YL, Maruyama J, Valerius O, Rizzoli SO, Ficner R, Irniger S, Braus GH (2012) The Aspergillus nidulans MAPK module AnSte11-Ste50-Ste7-Fus3 controls development and secondary metabolism. PLoS Genet 8: e1002816.

26. Hernández-Ortiz P, Espeso EA (2017) Spatiotemporal dynamics of the calcineurin target CrzA. Cell Signal 29: 168-180. 
27. Meyer V, Andersen MR, Brakhage AA, Braus GH, Caddick MX, Cairns TC, de Vries RP, Haarmann T, Hansen K, Hertz-Fowler C, et al. (2016) Current challenges of research on filamentous fungi in relation to human welfare and a sustainable bio-economy: a white paper. Fungal Biol Biotechnol 3: 6.

28. Lee M-K, Kwon N-J, Lee I-S, Jung S, Kim S-C, Yu J-H (2016) Negative regulation and developmental competence in Aspergillus. Sci Rep 6: 28874.

29. Oiartzabal-Arano E, Perez-de-Nanclares-Arregi E, Espeso EA, Etxebeste O (2016) Apical control of conidiation in Aspergillus nidulans. Curr Genet 62: 371-377.

30. Garzia A, Etxebeste O, Herrero-García E, Ugalde U, Espeso EA (2010) The concerted action of bZip and cMyb transcription factors FlbB and FlbD induces brlA expression and asexual development in Aspergillus nidulans. Mol Microbiol 75: $1314-1324$.

31. Kwon N-J, Garzia A, Espeso EA, Ugalde U, Yu J-H (2010) FlbC is a putative nuclear $\mathrm{C} 2 \mathrm{H} 2$ transcription factor regulating development in Aspergillus nidulans. Mol Microbiol 77: 1203-1219.

32. Etxebeste O, Ni M, Garzia A, Kwon NJ, Fischer R, Yu JH, Espeso EA, Ugalde U (2008) Basic-zipper-type transcription factor FlbB controls asexual development in Aspergillus nidulans. Eukaryot Cell 7: 38-48.

33. Pantazopoulou A, Peñalva MA (2009) Organization and dynamics of the Aspergillus nidulans Golgi during apical extension and mitosis. Mol Biol Cell 20: 4335-4347.

34. Garzia A, Etxebeste O, Herrero-Garcia E, Fischer R, Espeso EA, Ugalde U (2009) Aspergillus nidulans FlbE is an upstream developmental activator of conidiation functionally associated with the putative transcription factor FlbB. Mol Microbiol 71: 172-184.

35. Cortese MS, Etxebeste O, Garzia A, Espeso EA, Ugalde U (2011) Elucidation of functional markers from Aspergillus nidulans developmental regulator FlbB and their phylogenetic distribution. PLoS One 6: e17505.

36. Chu X-L, Feng M-G, Ying S-H (2016) Qualitative ubiquitome unveils the potential significances of protein lysine ubiquitination in hyphal growth of Aspergillus nidulans. Curr Genet 62: 191-201.

37. Mitchell AL, Attwood TK, Babbitt PC, Blum M, Bork P, Bridge A, Brown SD, Chang H-Y, El-Gebali S, Fraser MI, et al. (2018) InterPro in 2019: improving coverage, classification and access to protein sequence annotations. Nucleic Acids Res gky1100-gky1100.

38. Savoldi M, Malavazi I, Soriani FM, Capellaro JL, Kitamoto K, da Silva Ferreira ME, Goldman MHS, Goldman GH (2008) Farnesol induces the transcriptional accumulation of the Aspergillus nidulans Apoptosis-Inducing Factor (AIF)-like mitochondrial oxidoreductase. Mol Microbiol 70: 44-59. 
39. Arribere JA, Cenik ES, Jain N, Hess GT, Lee CH, Bassik MC, Fire AZ (2016) Translation readthrough mitigation. Nature 534: 719-723.

40. Taheri-Talesh N, Xiong Y, Oakley BR, Oakley B, Brakhage A (2012) The Functions of Myosin II and Myosin V Homologs in Tip Growth and Septation in Aspergillus nidulans. PLoS One 7: e31218.

41. Pantazopoulou A, Pinar M, Xiang X, Peñalva MA (2014) Maturation of late Golgi cisternae into RabE(RAB11) exocytic post-Golgi carriers visualized in vivo. Mol Biol Cell 25: 2428-2443.

42. Xiang X, Beckwith SM, Morris NR (1994) Cytoplasmic dynein is involved in nuclear migration in Aspergillus nidulans. Proc Natl Acad Sci 91: 2100-2104.

43. Peñalva MA, Zhang J, Xiang X, Pantazopoulou A (2017) Transport of fungal RAB11 secretory vesicles involves myosin- 5, dynein/dynactin/p25 and kinesin-1 and is independent of kinesin-3. 28: 947-961.

44. Markina-Iñarrairaegui A, Etxebeste O, Herrero-García E, Araújo-Bazán L, Fernández-Martínez J, Flores JA, Osmani SA, Espeso EA (2011) Nuclear transporters in a multinucleated organism: functional and localization analyses in Aspergillus nidulans. Mol Biol Cell 22: 3874-3886.

45. Abenza JF, Pantazopoulou A, Rodríguez JM, Galindo A, Peñalva MA (2009) Long-Distance Movement of Aspergillus nidulans Early Endosomes on Microtubule Tracks. Traffic 10: 57-75.

46. Wieser J, Adams TH (1995) flbD encodes a Myb-like DNA-binding protein that coordinates initiation of Aspergillus nidulans conidiophore development. Genes Dev 9: 491-502.

47. Spatafora JW, Aime MC, Grigoriev I V., Martin F, Stajich JE, Blackwell M (2017) The fungal tree of life: from molecular systematics to genome-scale phylogenies. Microbiol Spectr 5:.

48. Arratia-Quijada J, Sánchez O, Scazzocchio C, Aguirre J (2012) FlbD, a Myb Transcription Factor of Aspergillus nidulans, Is Uniquely Involved in both Asexual and Sexual Differentiation. Eukaryot Cell 11: 1132-1142.

49. Xiang X, Qiu R, Yao X, Arst HN, Peñalva MA, Zhang J (2015) Cytoplasmic dynein and early endosome transport. Cell Mol Life Sci 72: 3267-3280.

50. Son H, Kim M-G, Chae S-K, Lee Y-W (2014) FgFlbD regulates hyphal differentiation required for sexual and asexual reproduction in the ascomycete fungus Fusarium graminearum. J Microbiol 52: 930-939.

51. Matheis S, Yemelin A, Scheps D, Andresen K, Jacob S, Thines E, Foster AJ (2017) Functions of the Magnaporthe oryzae Flb3p and Flb4p transcription factors in the regulation of conidiation. Microbiol Res 196: 106-117.

52. Soufi A, Garcia MF, Jaroszewicz A, Osman N, Pellegrini M, Zaret KS (2015) Pioneer Transcription Factors Target Partial DNA Motifs on Nucleosomes to 
Initiate Reprogramming. Cell 161: 555-568.

53. Tahirov TH, Sato K, Ichikawa-Iwata E, Sasaki M, Inoue-Bungo T, Shiina M, Kimura K, Takata S, Fujikawa A, Morii H, et al. (2002) Mechanism of c-Myb$\mathrm{C} / \mathrm{EBP}$ beta cooperation from separated sites on a promoter. Cell 108: 57-70.

54. Cánovas D, Marcos AT, Gacek A, Ramos MS, Gutiérrez G, Reyes-Domínguez Y, Strauss J (2014) The histone acetyltransferase GcnE (GCN5) plays a central role in the regulation of Aspergillus asexual development. Genetics 197: 11751189.

55. Lind AL, Lim FY, Soukup AA, Keller NP, Rokas A (2018) An LaeA- and BrlAdependent cellular network governs tissue-specific secondary metabolism in the human pathogen Aspergillus fumigatus. mSphere 3: e00050-18.

56. Cove DJ (1966) The induction and repression of nitrate reductase in the fungus Aspergillus nidulans. Biochim Biophys Acta - Enzymol Biol Oxid 113: 51-56.

57. Käfer E (1965) Origins of translocations in Aspergillus nidulans. Genetics 52: 217 LP-232.

58. Galindo A, Calcagno-Pizarelli AM, Arst HN, Peñalva MÁ (2012) An ordered pathway for the assembly of fungal ESCRT-containing ambient $\mathrm{pH}$ signalling complexes at the plasma membrane. J Cell Sci 125: 1784-1795.

59. Etxebeste O, Herrero-García E, Araújo-Bazán L, Rodríguez-Urra AB, Garzia A, Ugalde U, Espeso EA (2009) The bZIP-type transcription factor FIbB regulates distinct morphogenetic stages of colony formation in Aspergillus nidulans. Mol Microbiol 73: 775-789.

60. Peñalva MA (2005) Tracing the endocytic pathway of Aspergillus nidulans with FM4-64. Fungal Genet Biol 42: 963-975.

61. Tilburn J, Scazzocchio C, Taylor GG, Zabicky-Zissman JH, Lockington RA, Davies RW (1983) Transformation by integration in Aspergillus nidulans. Gene 26: $205-221$.

62. Yang L, Ukil L, Osmani A, Nahm F, Davies J, De Souza CPC, Dou X, PerezBalaguer A, Osmani SA (2004) Rapid production of gene replacement constructs and generation of a green fluorescent protein-tagged centromeric marker in Aspergillus nidulans. Eukaryot Cell 3: 1359-1362.

63. Szewczyk E, Nayak T, Oakley CE, Edgerton H, Xiong Y, Taheri-Talesh N, Osmani SA, Oakley BR (2007) Fusion PCR and gene targeting in Aspergillus nidulans. Nat Protoc 1: 3111-3120.

64. Todd RB, Davis MA, Hynes MJ (2007) Genetic manipulation of Aspergillus nidulans: meiotic progeny for genetic analysis and strain construction. Nat Protoc 2: $811-821$.

65. Perez-de-Nanclares-Arregi E, Etxebeste O (2014) Photo-convertible tagging for localization and dynamic analyses of low-expression proteins in filamentous 
fungi. Fungal Genet Biol 70:.

66. Hervás-Aguilar A, Peñalva MA (2010) Endocytic machinery protein SlaB is dispensable for polarity establishment but necessary for polarity maintenance in hyphal tip cells of Aspergillus nidulans. Eukaryot Cell 9: 1504-1518.

67. Fernández-Martínez J, Brown C V, Díez E, Tilburn J, Arst HN, Peñalva MÁ, Espeso EA (2003) Overlap of Nuclear Localisation Signal and Specific DNAbinding Residues Within the Zinc Finger Domain of PacC. J Mol Biol 334: 667684.

68. Wortman JR, Gilsenan JM, Joardar V, Deegan J, Clutterbuck J, Andersen MR, Archer D, Bencina M, Braus G, Coutinho P, et al. (2009) The 2008 update of the Aspergillus nidulans genome annotation: a community effort. Fungal Genet Biol 46:.

69. Nayak T, Szewczyk E, Oakley CE, Osmani A, Ukil L, Murray SL, Hynes MJ, Osmani SA, Oakley BR (2006) A versatile and efficient gene-targeting system for Aspergillus nidulans. Genetics 172: 1557-1566. 


\section{Figure legends.}

2 Figure 1: Role of FlbE domains in the apical localization of FlbB.

3 A) Subcellular localization of GFP::FlbB in $g p d A^{\text {mini }}(\mathrm{OE}):: \mathrm{GFP}:: \mathrm{FlbB}$ (control),

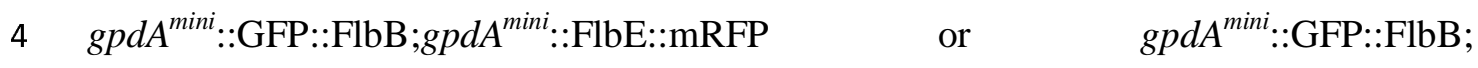

$5 \quad g p d A^{\min i}::$ FlbE::Stag strains. The graphs below show the intensity of green fluorescence

6 in the region delimited by the dotted arrows. The values show the fluorescence intensity

7 ratio between the tip and the most apical nucleus, as the average of 15 measurements for

8 each strain plus s.e.m. Scale bar $=5 \mu \mathrm{m}$

9 B) Phenotype of 1) GFP::FlbB;FlbE::mCh, 2) $g p d A^{\text {mini }}:$ GFP::FlbB, 3)

$10 \quad g p d A^{\text {mini }}::$ FlbE::mRFP and 4) $g p d A^{\text {mini }}::$ GFP::FlbB;gpd $A^{\text {mini }}::$ FlbE::mRFP strains after

1126 hours of culture in liquid AMM. The double-gpdA ${ }^{\text {mini }}$ strain developed conidiophores

12 (see the asterisk), which can be seen in the $3 \mathrm{x}$ amplification on the right. Scale bar $=10$

$13 \mu \mathrm{m}$.

14 C) Predicted functional domains within FlbE, based on the analyses described by [34]

15 (up) and [35] (down).

16 D) Phenotype of point $f l b E^{*}$ mutants in AMM plates after 72 hours of culture at $37^{\circ} \mathrm{C}$.

17 The graph shows conidia counts per $\mathrm{cm}^{2}$ for each mutant. Values are given as the mean

18 of three replicates plus s.e.m.

E) Subcellular localization of $g p d A^{\text {mini }}:$ GFP::FlbB (wild-type form) and

$20 g p d A^{m i n i}:: F l b E^{*}::$ RFP (mutant forms) in vegetative hyphae (the wild-type form is shown

21 as control). Scale bar $=5 \mu \mathrm{m}$. The graphs below show red and green fluorescence

22 intensities (arbitrary units) in hyphal segments covering the region between the tip and 
1 the most apical nucleus (asterisks indicate which hyphae have been analyzed). M:

2 merged.

3 F) Co-immunoprecipitation assays using bacterially expressed GST (negative control)

4 and GST::FlbB forms and crude extracts from strains expressing wild-type or mutant

$5 \quad g p d A^{m i n i}:: F l b E:: G F P$ forms W11A, K51A, D70A;D73A, Y85A;V86A and P182A.

6 Polyacrilamide gels stained with Coomassie blue are shown as controls.

$7 \quad$ See also Figure EV1.

\section{$9 \quad$ Figure 2: Analysis of FlbE::FlbB chimeras.}

10 A) Phenotype of wild-type (control), FlbE::GFP, FlbE ${ }^{(34-201)}:$ GFP and GFP::FlbE

11 strains in adequately supplemented AMM plates after 48 hours of culture at $37^{\circ} \mathrm{C}$. Scale

12 bar $=1 \mathrm{~cm}$.

13 B) Subcellular localization of FlbE::GFP, FlbE ${ }^{(34-201)}:$ GFP and GFP::FlbE chimeras in 14 vegetative hyphae. Asterisks indicate tips. Scale bar $=5 \mu \mathrm{m}$.

15 C) Immunoprecipitation assay using as baits $g p d A^{\text {mini }}$-driven endogenous FlbE::mRFP

16 (left) or FlbE ${ }^{(\mathrm{W} 11 \mathrm{~A})}:$ :mRFP (right) chimeras. The Western-blot below shows the levels of

17 GFP::FlbB in total extracts as well as non-retained (NR) and retained (R) fractions. A

18 Polyacrilamide gel stained with Coomassie blue is shown as control.

19 D) Phenotype of strains expressing $g p d A^{m i n i}$-driven (OE) FlbE::GFP (reference), FlbE ${ }^{(1-}$ $2{ }^{202)}::$ FlbB::GFP, FlbE ${ }^{(1-39)}:: F l b B:: G F P$ or FlbE ${ }^{(1-39 ; W 11 A)}:: F l b B:: G F P$ chimeras after 72

21 hours of culture at $37^{\circ} \mathrm{C}$ in MMA. Scale bar $=2 \mathrm{~cm}$. 
1 E) Subcellular localization of the above-mentioned chimeras in vegetative hyphae.

2 Asterisks indicate tips. Scale bar $=5 \mu \mathrm{m}$.

3 F) Phenotype of strains expressing T2A peptide-containing FlbB and FlbE chimeras

4 (wild-type or the G17A mutant) after 120 hours of culture in plates (diameter $=5.5 \mathrm{~cm}$ )

5 filled with AMM. Strains expressing 1) FlbB::GFP, 2) FlbE::mRFP and GFP::FlbB, or

6 3) FlbE::FlbB::GFP chimeras were used as controls. The graph on the right shows

7 conidia production (conidia $/ \mathrm{cm}^{2}$ ) for the strains on the left. Values for each strain and

8 time-point are given as the mean of three replicates plus s.e.m.

9 G) Fluorescence microscopy images corresponding to hyphae of strains expressing 1)

10 GFP::FlbB and FlbE::mRFP, 2) FlbE::FlbB::GFP, 3) FlbE::mRFP::T2A::FlbB::GFP

11 and 4) FlbE::mRFP::T2A ${ }^{(\mathrm{G} 17 \mathrm{~A})}::$ FlbB::GFP chimeras. White asterisks indicate hyphal

12 tips and arrows nuclei. The graphs on the right of each group of images show red and

13 green fluorescence intensities (arbitrary units) in hyphal segments covering the 14 indicated length. Scale bars $=5 \mu \mathrm{m}$.

15 See also Figure EV2

17 Figure 3: Role of FlbB Cysteines in the apical localization of FlbB and FlbE.

18 A) Diagram showing the position and conservation of the six cysteines within FlbB.

19 "Y" indicates conservation while " $\mathrm{X}$ " indicates no conservation. Based on Cortese et 20 al., $2011[35]$.

21 B) Phenotype of the strains expressing $g p d A^{\operatorname{mini}}$ (OE)-driven GFP::FlbB Cys-to-Ala 22 mutant chimeras, on AMM plates (diameter: $5.5 \mathrm{~cm}$ ) after 72 hours of culture at $37^{\circ} \mathrm{C}$. 
1 The graph on the right quantifies conidia produced by each strain (per $\mathrm{cm}^{2}$ ), as the mean

2 of three replicates plus s.e.m.

3 C) Subcellular localization of $g p d A^{\min i}$-driven mutant GFP::FlbB ${ }^{\text {(Cys-to-Ala) }}$ chimeras in

$4 \quad$ vegetative hyphae. Tips are indicated by asterisks. Scale bar $=5 \mu \mathrm{m}$.

5 D) Subcellular localization of GFP::FlbB and FlbE::mRFP in those FlbB ${ }^{\text {(Cys-to-Ala) }}$

6 mutants showing a fluffy phenotype (Cys272Ala. Cys382Ala, and

7 Cys272Ala;Cys382Ala). The reference wild-type strain and the Cys236Ala mutant were

8 used as controls. The graphs at the bottom correspond to hyphae indicated by an asterisk

9 and show green and red fluorescence intensity (arbitrary units) along hyphal segments

10 covering the tip and the most apical nucleus. Scale bar $=5 \mu \mathrm{m}$.

11 E) Co-immunoprecipitation assays between bacterially-expressed GST::FlbB forms

12 (wild-type and those Cys-to-Ala mutants causing a fluffy phenotype) and crude protein

13 extracts from a strain expressing FlbE::GFP. NR: Not-retained fraction. R: Retained

14 fraction. The graph on the right shows the intensity ratios between western-blot and

15 coomassie bands for each assay. The values are the mean of three replicates plus s.e.m.

16 See also Figure EV3.

18 Figure 4: MyoE and NudA in the subcellular localization of FlbB:

19 A) Subcellular localization of a $g p d A^{\text {mini }}$-driven GFP::FlbB chimera in a medium

20 containing Latrunculin B $(100 \mu \mathrm{m})$ as an inhibitor of actin filament polymerization. See

21 reference [23]. 
1

2 The deletion of $m y o E$ causes the spreading of GFP::FlbB into an apical crescent instead

3 of accumulating at the apex [41]. Microfilament-like structures decorated with

4 GFP::FlbB were also observed. The graphs show the intensity of green fluorescence

5 along the segments indicated by the red and blue dotted lines. In all panels, scale bar $=5$

$6 \mu \mathrm{m}$.

7 C) GFP::FlbB dynamics in three vegetative hyphae of strains co-expressing FlbE::RFP,

8 both under the control of $g p d A^{\min i}(\mathrm{OE})$. The six frames shown in the left group of

9 images were selected from Video EV4A. The dotted line in the first frame indicates the

10 region analyzed in the kymograph below, which shows patches of GFP::FlbB moving in

11 acropetal and basipetal directions between the tip and the most apical nucleus (number

12 1). Number 2 indicates a subapical, motionless spot of GFP::FlbB from which basipetal

13 patches depart in frames corresponding to 4150 and $4400 \mathrm{~ms}$ (number 4). Finally,

14 number 3 indicates filamentous structures that apparently link the subapex and the apex.

15 The remaining two hyphae (middle and right) correspond to Videos EV4B and EV4C.

16 Scale bars $=5 \mu \mathrm{m}$.

D) Subcellular localization of GFP::FlbB in wild-type and nudA1 genetic backgrounds

at 28 or $37^{\circ} \mathrm{C}$. The graphs show the intensity of green fluorescence along the hyphal segments covered by the dotted lines. The ratios between the intensity of fluorescence at

20 the tip and the most apical nucleus are also included. The dotted red square indicates the

21 loss of nuclear localization of FlbB when NudA activity is inhibited (nudAl background at $\left.37^{\circ} \mathrm{C}\right)$. Scale bars $=5 \mu \mathrm{m}$.

See also Video EV4. 


\section{$1 \quad$ Figure 5: Role of FlbD in the nuclear accumulation of FlbB.}

2 A) Left: Subcellular localization of an FlbB::GFP chimera (driven by the native

3 promoter) in vegetative hyphae of wild-type (up) and $\Delta f l b D$ strains (down). In the latter

4 background, nuclei were marked using a HhoA::mCh chimera. Asterisks indicate tips

5 while the arrows indicate nuclei. The graphs on the right show green and red

6 fluorescence intensity (arbitrary units) along the dotted lines. The ratios between the

7 intensity of fluorescence at the tip and the most apical nucleus are also included.

8 Middle: Cytoplasmic movement of FlbB::GFP in a $\triangle f l b D$ genetic background. A hypha

9 with mobile fluorescent FlbB::GFP patches is shown. The kymograph and the diagram

10 below analyze the acropetal (red arrow) and basipetal (black arrows) movement of those

11 patches along the dotted line. Right: Analysis of the dynamics of FlbB::GFP patches in

12 the same strain and in a medium containing benomyl as a MT-destabilizing agent.

13 Vertical lines in the kymograph and the diagram indicate an inhibition of FlbB::GFP

14 movement as result of the addition of the drug. In all panels, scale bar $=5 \mu \mathrm{m}$.

15 B) Domain analysis of FlbD sequence. The position and length of each of the five

16 predicted domains, as well as their general conservation in orthologs of specific species

17 within Eurotiomycetes (Eurotiales and Onygenales) and Sordariomycetes, is shown.

18 " $\mathrm{Y}$ " indicates conservation while " $\mathrm{X}$ " indicates no conservation. The alignment of all

19 the orthologs of FlbD analyzed in this work can be seen in Appendix Figure S1. The

20 position of the mutations within domains D1 (cMyb) and D5 (a predicted

21 LIG_NRBOX) characterized in panels $\mathrm{C}$ andD is also indicated.

22 C) Phenotype of wild-type and null flbD strains, and strains expressing mutant

$23 \mathrm{FlbD}^{(\mathrm{E} 14 \mathrm{G} ; \mathrm{R} 87 \mathrm{Q})}, \mathrm{FlbD}^{(1-112)}$ or $\mathrm{FlbD}^{(\mathrm{L} 309 \mathrm{~A} ; \mathrm{L} 312 \mathrm{~A})}$ forms in $\mathrm{AMM}$ plates (diameter $=5.5 \mathrm{~cm}$ ) 
1 after 72 hours of culture. The graph on the right shows conidia production per $\mathrm{cm}^{2}$ for

2 each strain at this time-point. Values given are the mean of three replicates plus s.e.m.

3 D) Subcellular localization of GFP::FlbB in the $f l b D$ mutant backgrounds characterized

4 in panel C. Asterisks indicate hyphal tips and arrowheads, nuclei. Scale bar $=5 \mu \mathrm{m}$.

5 E) Phenotype of a strain expressing a GFP::FlbB chimera bearing a double Leu-to-Ala

6 substitution in positions 333 and 334, after 72 hours of culture in AMM and compared

7 to reference GFP::FlbB and $\Delta f l b B$ strains. Diameter of plates is $5.5 \mathrm{~cm}$. The graph

8 shows conidia production per $\mathrm{cm}^{2}$ for each strain at the same time-point. Values given

9 are the mean of three replicates plus s.e.m.

10 F) Subcellular localization of wild-type and (L333A; L334A) GFP::FlbB chimeras in

11 vegetative hyphae. Asterisks and the arrowhead indicate hyphal tips and a nucleus,

12 respectively. Scale bar $=5 \mu \mathrm{m}$.

13 See also Figure EV5.

15 Figure 6: Working model for FlbB dynamics in hyphae of $A$. nidulans.

16 A) Diagram showing the morphology of a hypha, including a branch, a septum (rings

17 that separate cells within hyphae), nuclei and an actively growing tip.

18 B) Magnification of the region between a tip and its closest nucleus, showing a general

19 model for the acropetal and basipetal transport of FlbB. Each player is indicated below.

20 Dotted orange squares mark the features that are analyzed in more detail in the

21 following panels. 
1 C) Two hypothetic configurations of the signaling complex formed by FlbB and FlbE,

2 1:1 (left) or 2:2 (right). In both of them, FlbB interacts with FlbE through the bZIP

3 domain. At least five domains of $\mathrm{FlbE}$ (E1, E4, the linker L region, E5 and, to lower

4 extent, E3) would participate in the interaction with the bZIP of FlbB (see also [34]). At

5 least domain E1 would link the complex to the corresponding transport pathway, maybe

6 a vesicle attached to a still unknown molecular motor. Cys382 [23] and Cys272 of FlbB

7 are required for the acropetal transport of the FlbB/FlbE complex but not for their

8 interaction. They could mediate an interaction with the transport vesicle or be required

9 in order to acquire a specific three-dimensional conformation essential to join the

10 transport pathway (this last option is not considered in the model). Finally, myosin V,

11 MyoE, would transport the complex from the subapex to the growing apex of the tip.

12 D) At the hyphal subapex, FlbB would join a dynein-mediated basipetal transport

13 pathway (probably attached to a vesicle or an early-endosome) that would approximate

14 the TF to the nucleus. A NLS and a LxxLL motif are required for the import of FlbB

15 across nuclear pores and its accumulation in nuclei (see also [23]), as well as the cMyb

16 domain and an additional LxxLL motif of FlbD (see panel E).

17 E) Both FlbB and FlbD bind a common region of 300 bp within the promoter of $\operatorname{brlA}$,

$18 \operatorname{brlA}^{p}$ [30]. Based on previous publications describing the interaction of cMyb and bZIP

19 proteins for the control of gene expression [53], a heterodimerization model is proposed

20 on the left. Considering the essentiality of FlbD for the nuclear accumulation of FlbB,

21 as well as the similarities between cMyb (transcriptional regulation) and SANT

22 (chromatin remodeling) domains (http://www.aspergillusgenome.org/cgi-

23 bin/protein/proteinPage.pl?dbid=ASPL0000052812\&seq_source=A.\%20nidulans\%20F

24 GSC\%20A4), a more speculative "Remodeling + Heterodimerization" model is

25 proposed. In this model, FlbD binds $b r l A^{p}$ first, inducing, maybe in combination with 
1 other proteins such as GenE or LaeA [54,55], a chromatin remodeling event that enables

2 heterodimerization with FlbB and binding of both TFs to their targets at $b r l A^{\mathrm{p}}$. The NLS

3 of FlbB improves DNA binding [59] and the LxxLL motifs of both FlbD and FlbB may

4 play a modulatory role. 


\section{Supplementary material.}

2 Appendix Table S1: Enrichment of the LxxLL motif in transcription factors of $A$.

3 nidulans. To analyze if the LxxLL motif is enriched in TFs of A. nidulans compared to

4 other GO terms, the work by Wortman and colleagues was taken as a reference [68].

5 The authors predicted the presence of 490 TF-coding genes in A. nidulans, a 4,6 \% of a

6 total of 10,701 protein-coding genes. A total of 2,227 A. nidulans proteins containing at

7 least one LxxLL motif were identified. FlbB and FlbD were the only known TFs

8 controlling conidiation with such a motif in their amino acid sequences. The table

9 compares the number of LxxLL motif-containing proteins with GO terms associated

10 with TFs, such as Transcription, Nucleus, Nuclear or Transcription factor, with the total

11 number of proteins associated to those GO terms in the A. nidulans proteome. The

12 enrichment is calculated as the ratio between these parameters, and compared to other

13 GO terms not directly associated with TFs, such as translation, mitochondria, activity

14 and metabolic.

15 Appendix Table S2: Oligonucleotides used in this study.

16 Appendix Table S3: Strains used in this study.

18 Figure EV1: FlbE analysis.

19 A) Procedure followed for the generation of a dual-OE strain co-expressing GFP::FlbB

20 and FlbE::GFP, both driven by $g p d A^{\min i}$. The promoter of $f l b E, g p d A^{\min i}, f l b E$ coding

21 region, RFP or Stag plus the pyrG gene from A. fumigatus, and the 3'-UTR region were

22 amplified independently and fused. The amplicon was used to transform protoplasts of a 
1 strain expressing GFP::FlbB driven by $g p d A^{\min i}$. Recombination was induced at the $f l b E$

2 locus.

3 B) Phenotype of the strains analyzed in Figure 1A after 72 hours of culture in solid

$4 \mathrm{AMM}$ at $37{ }^{\circ} \mathrm{C}$ (diameter of plates is $5.5 \mathrm{~cm}$; OE: $g p d A^{\operatorname{mini}}$-driven). The graph below

5 quantifies conidia production for each strain (control: $1.56 \times 10^{7} \pm 0.6 \times 10^{7}$ conidia /

$6 \quad \mathrm{~cm}^{2}$; mRFP-tagged strain: $1.2 \times 10^{7} \pm 0.3 \times 10^{7}$ conidia $/ \mathrm{cm}^{2}$; S-tagged strain: $1.35 \mathrm{x}$

$7 \quad 10^{7} \pm 0.1 \times 10^{7}$ conidia $/ \mathrm{cm}^{2}$ ), calculated as the average value of three replicates plus 8 s.e.m.

9 C) Strategy followed for the generation of strains expressing $g p d A^{\text {mini }}$-driven mutant 10 FlbE*::GFP strains. Using oligonucleotides bearing the designed flbE mutations, two

11 PCR fragments were synthesized and fused. The fusion construct was used to transform 12 protoplasts of a wild-type, TN02A3 [69], strain.

13 D) Subcellular localization of $g p d A^{m i n i}$-driven wild-type and mutant FlbE::GFP

14 chimeras in vegetative hyphae. Asterisks indicate hyphal tips. Scale bar $=5 \mu \mathrm{m}$.

15 E) Subcellular localization of GFP::FlbB (driven by the native promoter) in a strain that 16 expresses constitutively a mutant $\mathrm{FlbE}^{(\mathrm{D} 70 \mathrm{~A} ; \mathrm{D} 73 \mathrm{~A})}:$ :mRFP chimera. The asterisk indicates 17 the tip. Scale bar $=5 \mu \mathrm{m}$.

18 F) Immunodetection of $g p d A^{\min i}(\mathrm{OE})$-driven wild-type and mutant FlbE::GFP chimeras 19 in crude extracts of the corresponding strains. The Coomassie-stained gel is shown as a 20 loading control.

21 See also Figure 1. 


\section{Figure EV2. Analysis of E1 domain of FlbE.}

2 A) and B) Procedures developed for the generation of strains expressing GFP::FlbE (A)

3 or $\mathrm{FlbE}^{(34-202)}:$ GFP (B) chimeras, both driven by the native flbE promoter. Cassettes

4 were fused and used to transform, respectively, protoplasts of a $\Delta f l b E$ or a wild-type

5 strain. Selection of transformants in panel A was done using fluororotic acid $(2 \mathrm{mg} / \mathrm{mL})$

6 [32].

7 C) Immunoprecipitation assay using bacterially expressed GST (negative control) and

8 GST::FlbE forms as bait and a crude protein extract of a strain expressing an

9 FlbB::HA $\mathrm{HA}_{3 \mathrm{x}}$ chimera. A polyacrilamide gel stained with Coomassie blue shows the 10 concentration of each bait.

11 D) and E) Procedure developed for the generation of strains expressing $g p d A^{\text {mini }}$-driven

12 FlbE::FlbB::GFP (D), as well as FlbE ${ }^{(1-39)}:: F l b B:: G F P$ and FlbE $^{(1-39 ; \mathrm{W} 11 \mathrm{~A})}:: F l b B:: G F P$

13 chimeras (E). Cassettes for transformation were assembled by the fusion-PCR technique

$14[62]$ and all of them were used to transform protoplasts of a $\Delta f l b B$ strain.

15 Recombination was induced at the flbE locus, in order to guarantee that the only source

16 of FlbE and FlbB was the one derived from the translation of the constructs.

17 F) Western-blot for the detection of the chimeras described in panels D and E in crude 18 protein extracts of the corresponding strains. Polyacrilamide gels stained with 19 Coomassie blue are shown as loading controls.

20 G) Procedure developed for the generation of strains expressing $g p d A^{\operatorname{mini}}$-driven

21 FlbE::mRFP::T2A::FlbB::GFP or FlbE::mRFP::T2A ${ }^{(\mathrm{G} 17 \mathrm{~A})}:$ :FlbB::GFP chimeras. Again,

22 cassettes for transformation were assembled by the fusion-PCR technique [62] and all

23 of them were used to transform protoplasts of a $\Delta f l b B$ strain. Recombination was 
1 induced at the flbE locus, in order to guarantee that the only source of FlbE and FlbB

2 was the one derived from the translation of the constructs.

$3 \mathrm{H})$ Western-blot experiment for the determination of the efficiency of the wild-type or

4 mutant (G17A) T2A viral peptide in Aspergillus nidulans. Two peptides, FlbE::mRFP

$5(47 \mathrm{kDa})$ and FlbB::GFP $(73 \mathrm{kDa})$ were detected when the wild-type T2A peptide was

6 used, together with the full length FlbE::mRFP:T2A::FlbB::GFP chimera (120 kDa).

7 Only this latter form was detected when the mutant G17A T2A form was used. Extracts

8 of strains expressing 1) FlbE:FlbB::GFP (96 kDa), or 2) FlbB::mRFP (47 kDa) and

9 FlbB::GFP (73 kDa) chimeras were used as controls.

\section{Figure EV3. Mutagenesis of FlbB cysteines.}

12 A) Procedure used for the generation of $g p d A^{\min i}$-driven GFP::FlbB ${ }^{\text {(Cys-to-Ala) }}$ mutants

13 [23]. A plasmid containing the fusion $g p d A^{m i n i}:: g f p:: f l b B_{c D N A}$ was mutagenized by PCR

14 using appropriate mutant oligonucleotides. The presence of the desired mutation was

15 confirmed by sequencing and the mutant plasmid was used to transform protoplasts of a

$16 \Delta f l b B$ strain. Selection of transformants was done based on the $p y r o A^{+}$phenotype and

17 the correct recombination was confirmed by Southern-blot. Asterisks indicate the

18 substitution of a codon coding for a Cys by a mutant codon coding for an Ala.

19 B) Western-blot showing that all Cys mutants expressed GFP::FlbB ${ }^{\text {(Cys-toAla) }}$ chimeras of

20 the same size. Strains expressing one or two copies of the mutant plasmid were

21 compared. The coomassie-stained gel is shown as a loading control.

22 See also Figure 3. 
2 Videos EV4: Subcellular dynamics of FlbB at hyphal tips. Videos corresponding to

3 three hyphae of the dual-OE strain expressing GFP::FlbB and FlbE::mRFP both driven

4 by the $g p d A^{m i n i}$ promoter. Time intervals for each frame are $0.222(\mathrm{~A}), 0.400$ (B) and

$50.200(\mathrm{C})$ seconds, respectively. Videos were generated using ImageJ (10 frames per

6 second).

7 See also Figure 4.

\section{$9 \quad$ Figure EV5: Role of FlbD in the nuclear accumulation of FlbB.}

10 A) Conidiophore development in liquid MMA as a result of $f l b D$ overexpression.

11 Phenotypes of strains 1) FlbB::GFP, 2) $a l c A^{p}:: f l b D$ and 3) $a l c A^{p}:: f l b D$; FlbB::GFP are

12 shown after 18 hours of culture in MMA (2\% glucose was used as the carbon source;

13 repression of $a l c A^{p}$ ) (left column) or additional 22 hours of culture in MMA that 14 contained threonine $(100 \mathrm{mM})$ as the carbon source (induction of alc $A^{p}$; middle column

15 and 2.5x magnifications on the right). Scale bar $=25 \mu \mathrm{m}$. The control strain generated a

16 limited number of conidiophores when threonine was used but those structures included

17 only 1-3 phialides and a limited number of conidia in each phialide, resembling the

18 morphology described by Skromne and colleagues for the simplified conidiophores

19 produced by $A$. nidulans under carbon starvation conditions [19]. alc $A^{p}:$ flbD strains

20 developed complex conidiophores.

21 B) Effect of flbD overexpression on solid culture medium. 1) FlbB::GFP, 2)

$\left.22 a l c A^{p}:: f l b D, 3\right) a l c A^{p}:: f l b D$; FlbB::GFP and 4) $\Delta f l b D$ strains were cultured under

23 repressing (ACM using 2\% glucose as the carbon source) (upper line) or purportedly 
1 inducing conditions (ACM plus $1 \%$ ethanol or $100 \mathrm{mM}$ threonine) (middle and lower

2 lines) for 72 hours at $37^{\circ} \mathrm{C}$. Diameter of plates is $5.5 \mathrm{~cm}$. Diagrams on the right show

3 conidia production by each strain. The values given are the mean of three replicates plus

4 s.e.m.

5 C) Subcellular localization of FlbB::GFP in vegetative hyphae of wild-type (up, left),

$6 \Delta f l b D$ (bottom, left), or $a l c A^{p}:: f l b D$ (right) strains in liquid AMM. alc $A^{p}$ was repressed

7 by supplementing the medium with glucose (0.1\%; up) and induced by adding

8 threonine (100 mM; bottom), respectively. The graphs show fluorescence intensity

9 along the dotted lines. Scale bar $=5 \mu \mathrm{m}$.

10 D) Phenotype of reference wild-type and null flbD strains, and strains expressing

11 FlbD::GFP, FlbD::HA $3 x$, HA $3 x:: F l b D$ or $H_{3 x}:: F l b D:: G F P$ chimeras in AMM plates

12 (diameter: $5.5 \mathrm{~cm}$ ) after 48 (upper row) and 72 (lower row) hours of culture at $37^{\circ} \mathrm{C}$.

13 The graph on the right shows conidia production by each strain after 48 hours of

14 culture. Values given are the mean of three replicates plus s.e.m.

15 E) Immunodetection of FlbD::HA $3 x$, FlbD::GFP, HA $\mathrm{H}_{3 x}:: F l b D$ and $\mathrm{HA}_{3 \mathrm{x}}:$ FlbD::GFP

16 chimeras. The coomassie-stained gel is shown as a loading control.

17 See also Figure 5.

19 Appendix Figure S1: Sequence alignment of FlbD orthologs, obtained using the

20 Genedoc software (version 2.7.000). Green boxes indicate the extension of the FlbD

21 regions conserved in the orthologs of species from the Eurotiales order. Those domains

22 that are conserved in all orthologs $(\mathrm{N}-$ and $\mathrm{C}$-terminal domains) are in red.

23 Nomenclature: Aory, Aspergillus oryzae; Afla, A. flavus; Anig, A. niger; Ater, A. 
1 terreus; Acla, A. clavatus; Afum, A. fumigatus, Nfis, Neosarotya fischeri; Tsti,

2 Talaromyces stipitatus; Ptri, Pyrenophora tritici; Sscl, Sclerotinia sclerotorium; Tver,

3 Trichophyton verrucosum; Aben, Arthroderma benhamiae; Trub, T. rubrum; Cpos,

4 Coccidioides posadasii; Cimm, C. immitis; Ader, Ajellomyces dermatitidis; Pbra,

5 Paracoccidioides brasiliensis; Fpse, Fusarium pseudograminearum; Foxy, F.

6 oxysporum; Fver, F. verticillioides; Vdah, Verticillium dahliae; Ndis, Neurospora

7 discreta; Smac, Sordaria macrospora; Ncra, N. crassa; Ntre, N. tetrasperma. 
bioRxiv preprint doi: https://doi.org/10.1101/467563; this version posted December 18, 2018. The copyright holder for th A not certified by peer review) is the author/funder, who has franted bioRxiv a license to display the preprint in perpetuity OEOFlbB

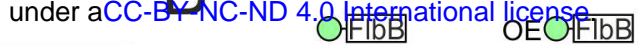
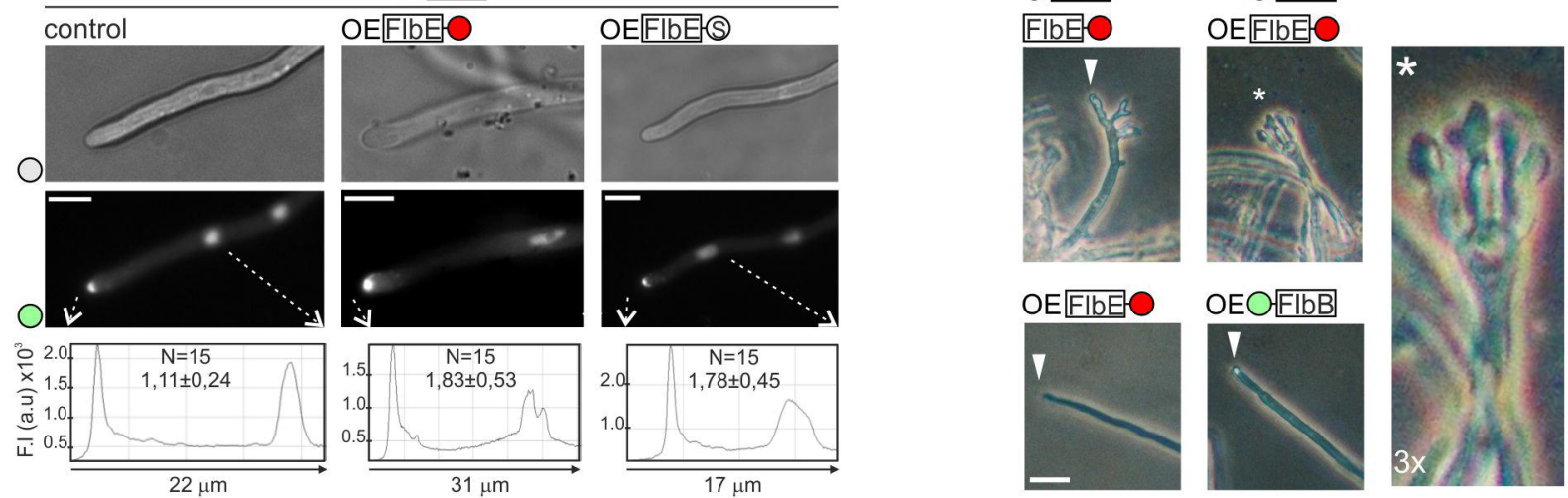

C
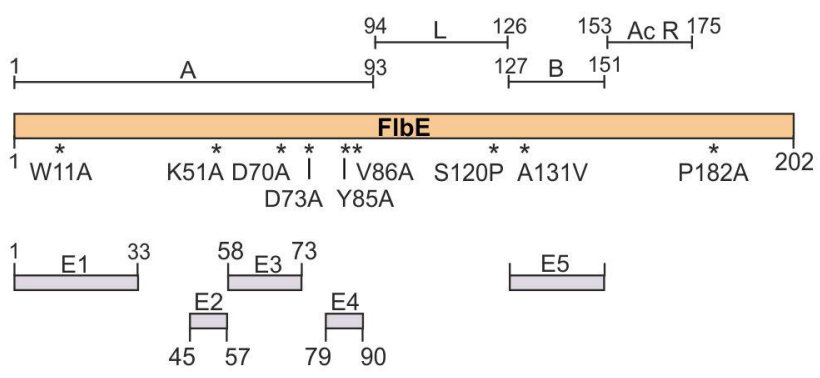

D

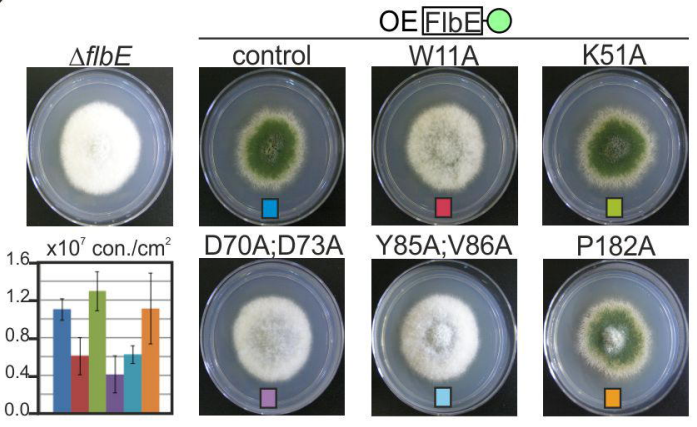

E OEFIbE
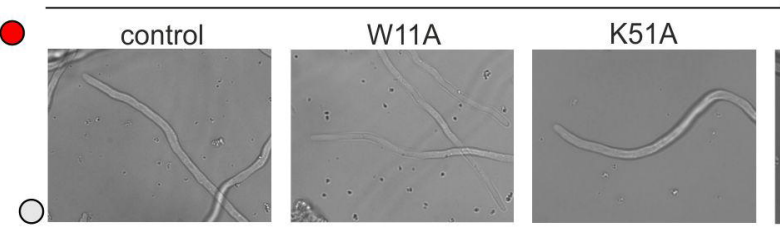

OEOFlbB
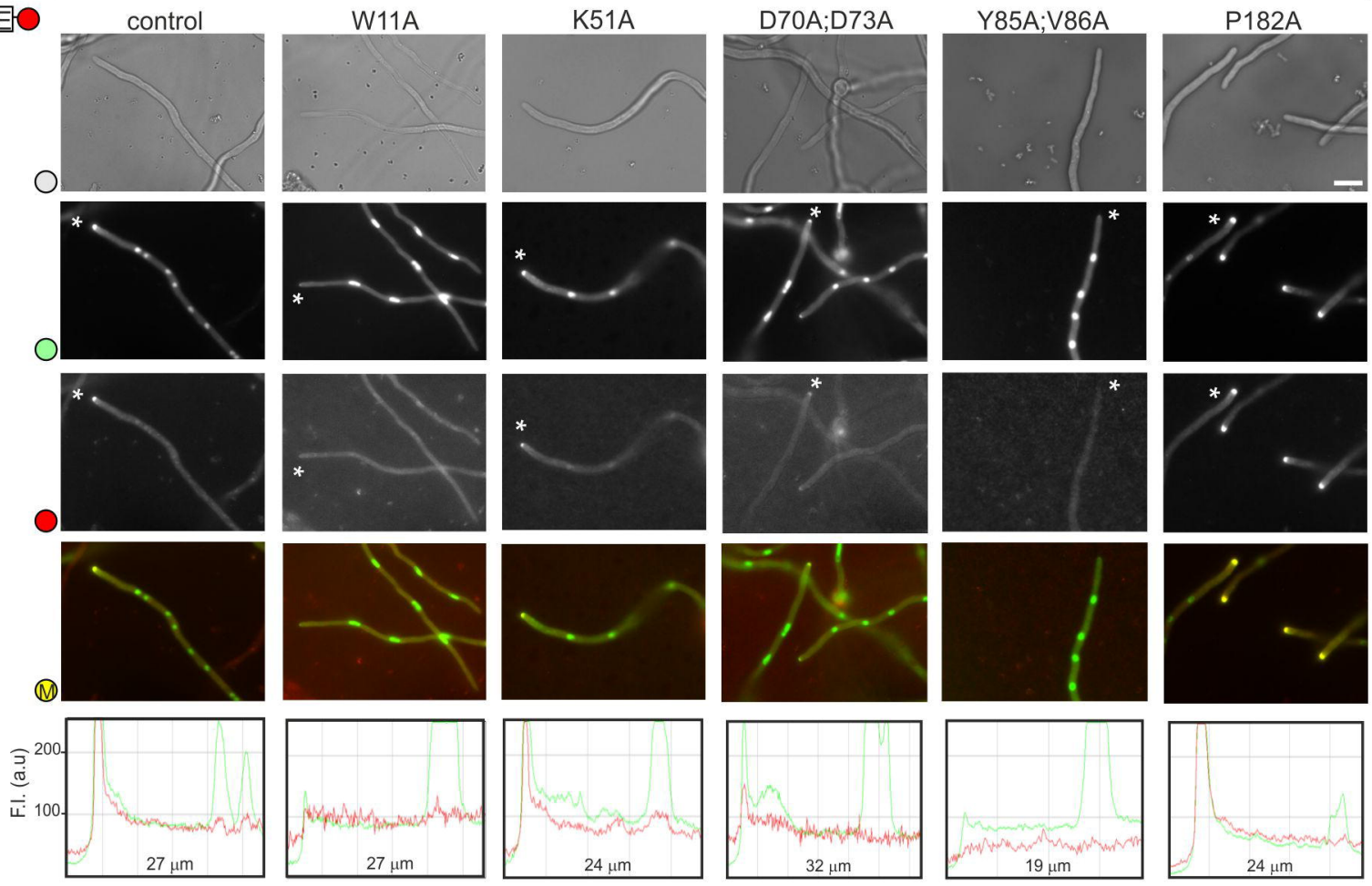

$\mathrm{F}$
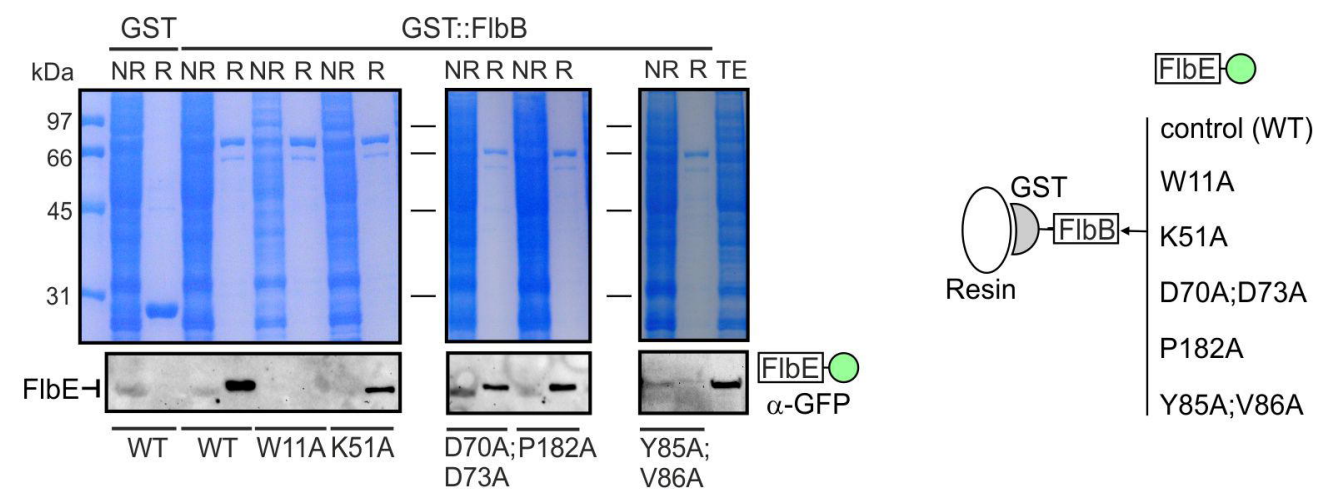
A $\quad$ B

Control

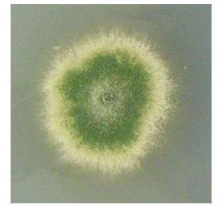

FlbErO

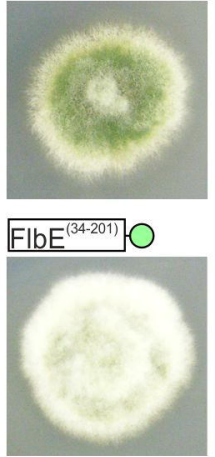

OFlbE

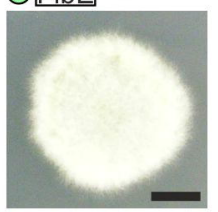

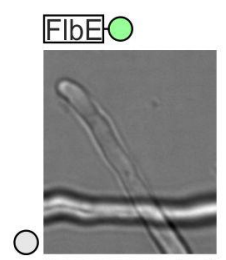

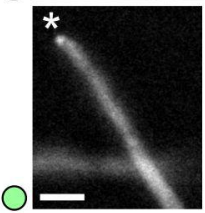

C

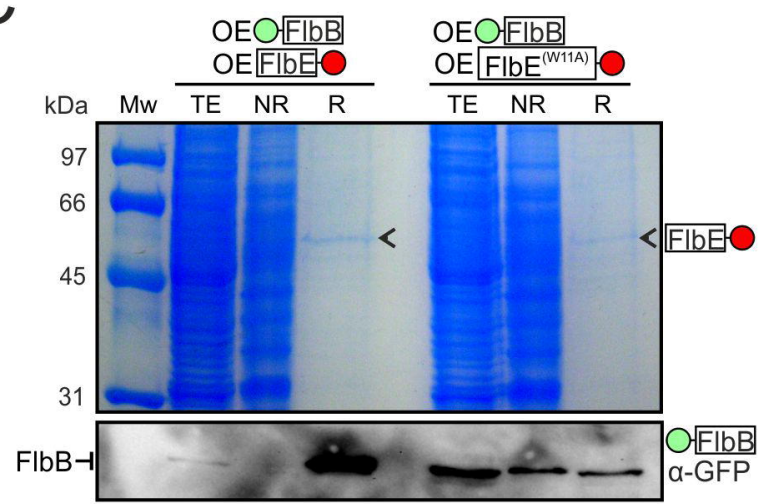

$\mathrm{FlbE}^{(34-201)} \mathrm{O}$
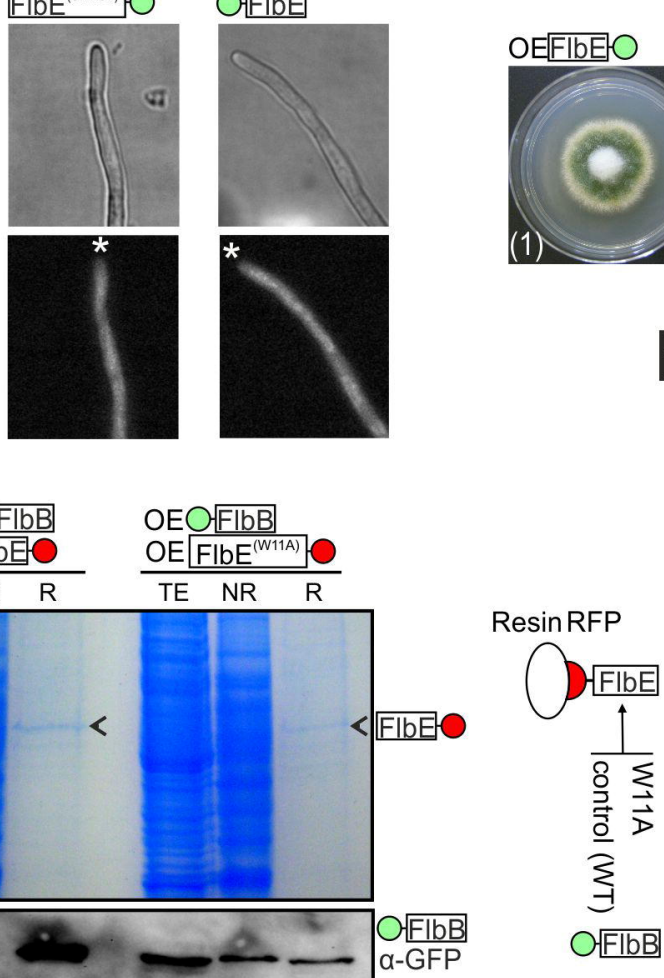

OE FlbE::FlbB
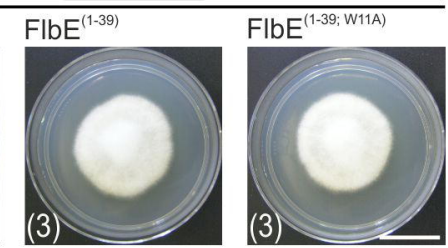

E

OEFlbE::FlbB

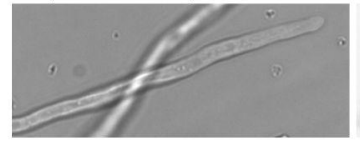

$\mathrm{OE} \mathrm{FlbE}^{(1-39)}: \mathrm{FlbB} \mathrm{O}$
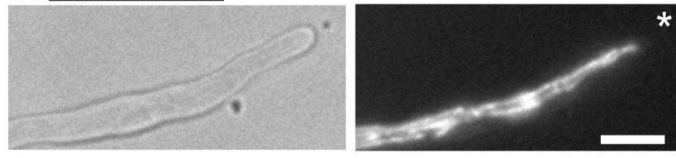

$\mathrm{OE} \mathrm{FlbE}^{(1-39 ; \mathrm{W} 11 \mathrm{~A})} .: \mathrm{FlbB} O$
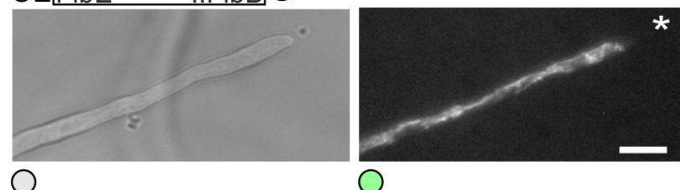

E

120 hours of culture in AMM

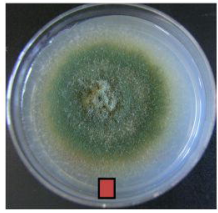

$\mathrm{FlbB} O$

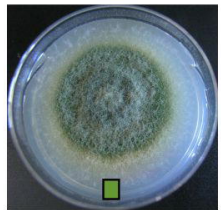

OEOFlbB

OEFIbEO

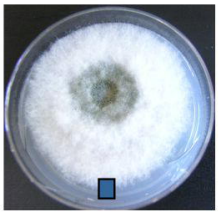

OEFIbE::FlbB

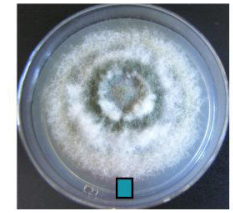

T2A

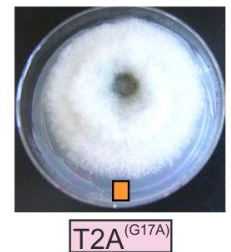

OEFIbEOTHFIbBO

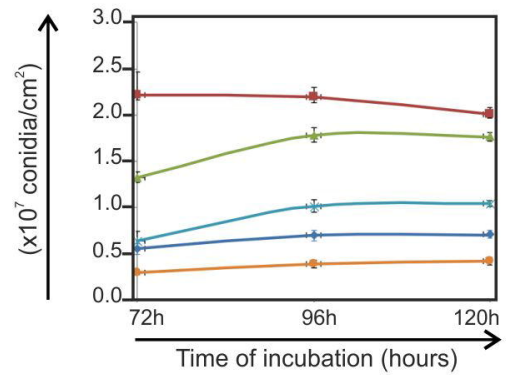

$G$
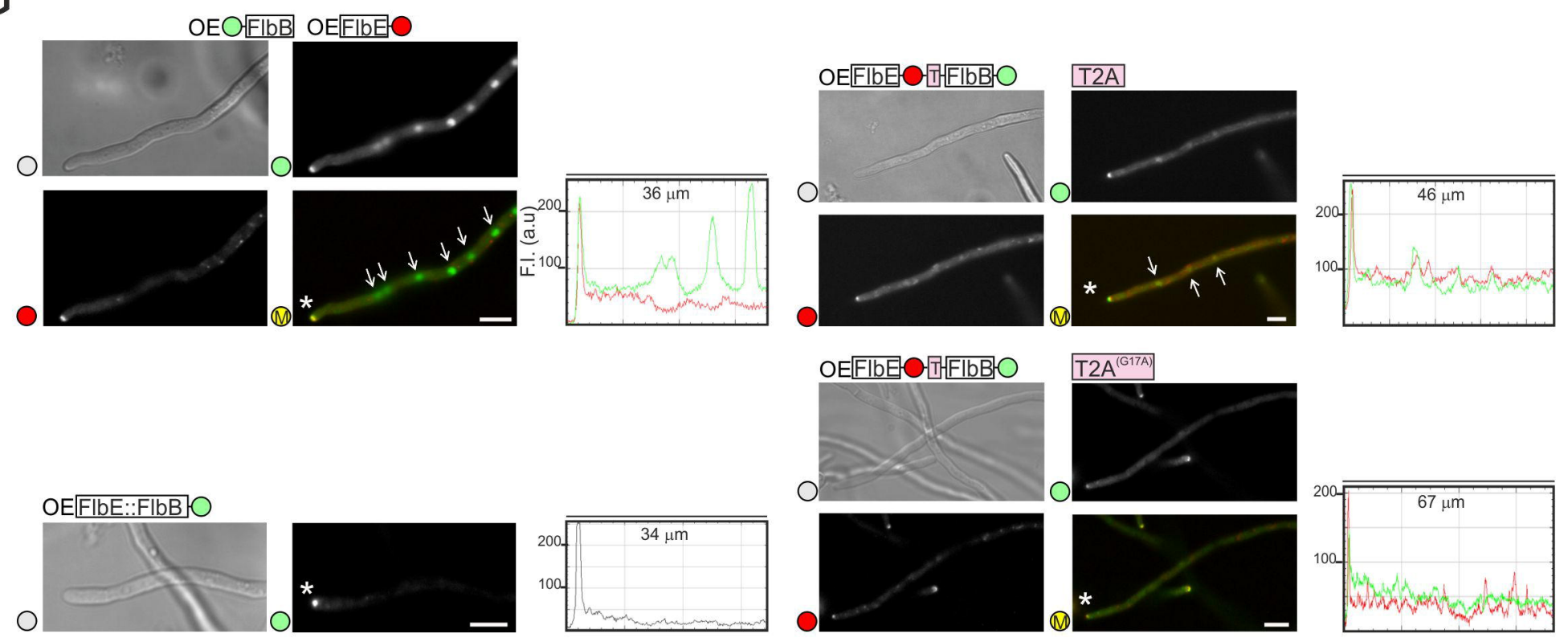

OEFlbE::FlbB $O$

o

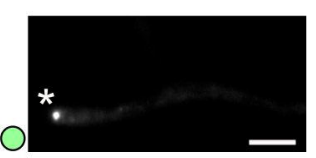

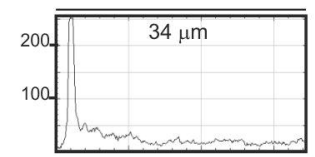


B

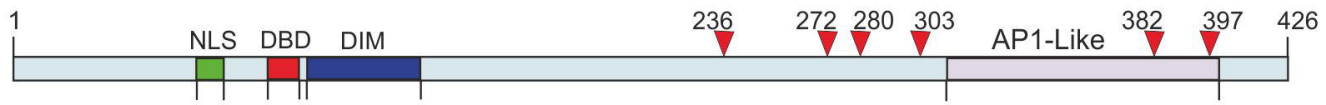

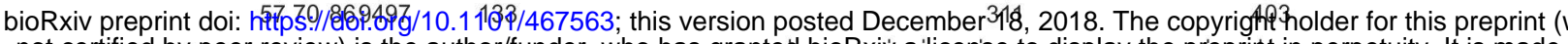
not certified byxhPferg reviews. is the author/funder, who has granted bioRxiv a :license to display the preprint in perpetuity. It is made

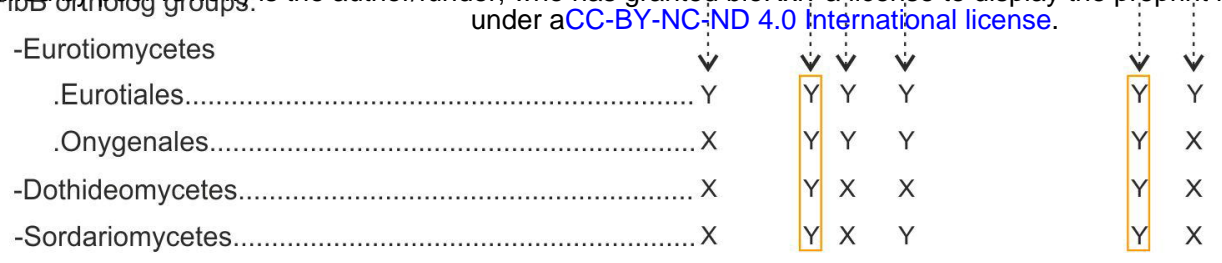

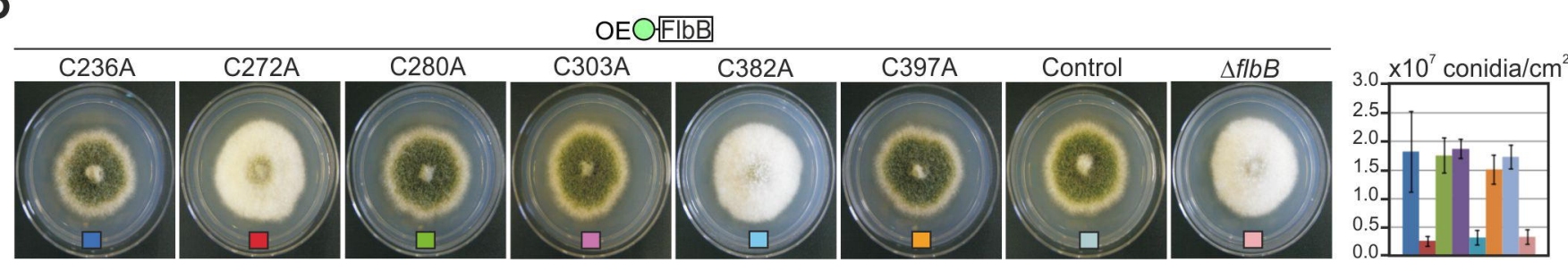

C

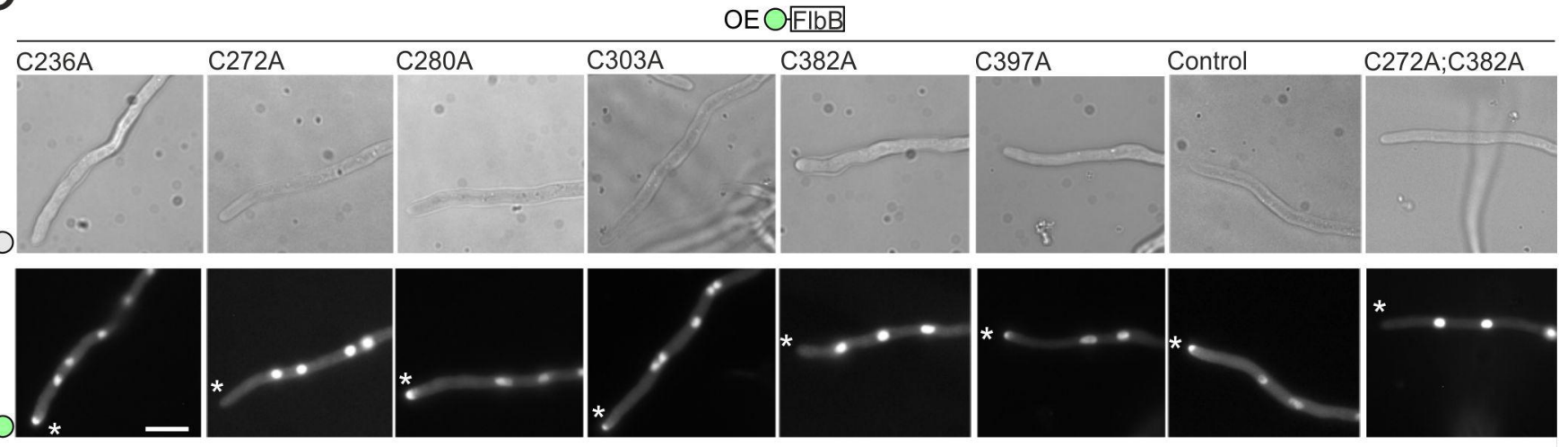

D

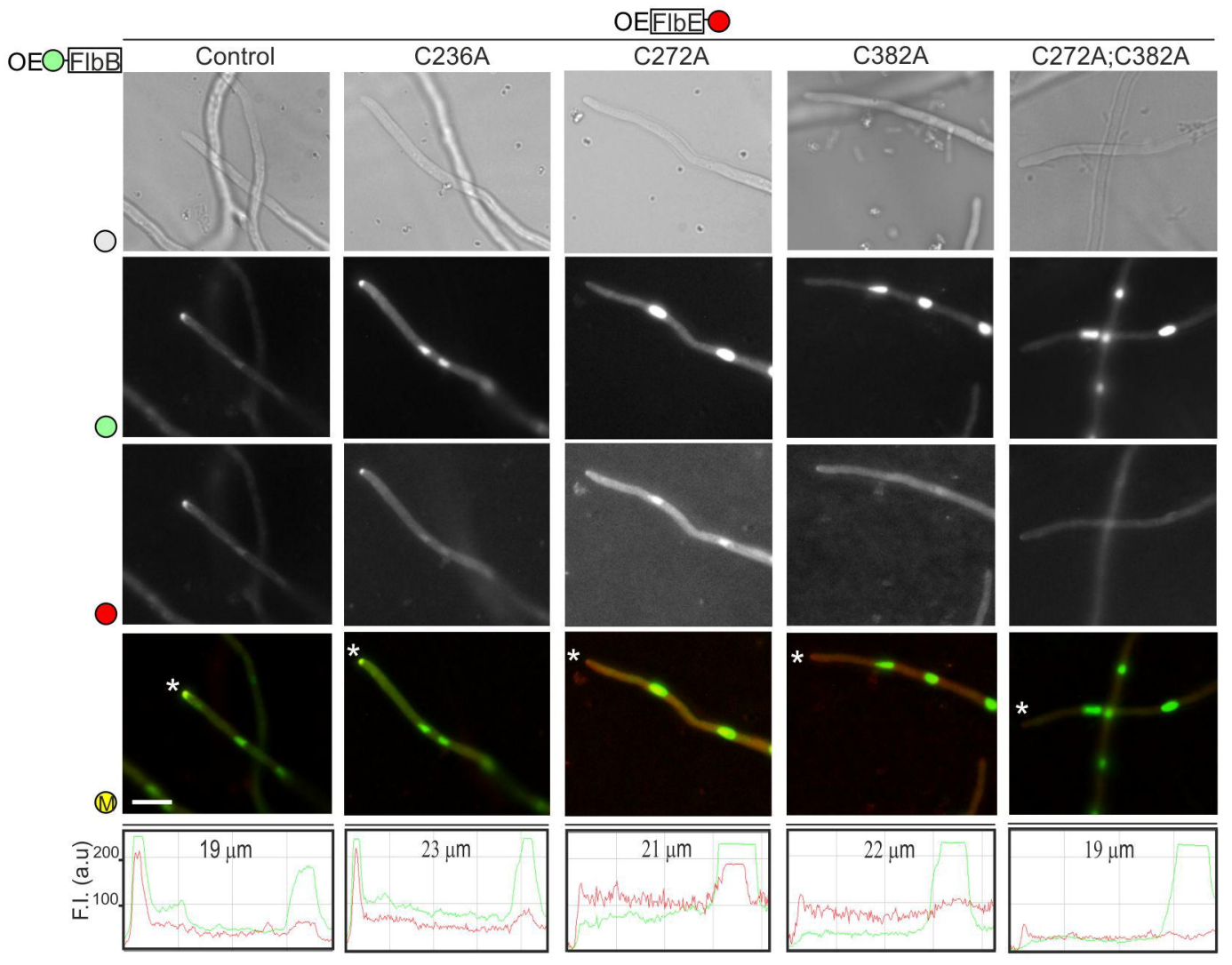

GST::FlbB

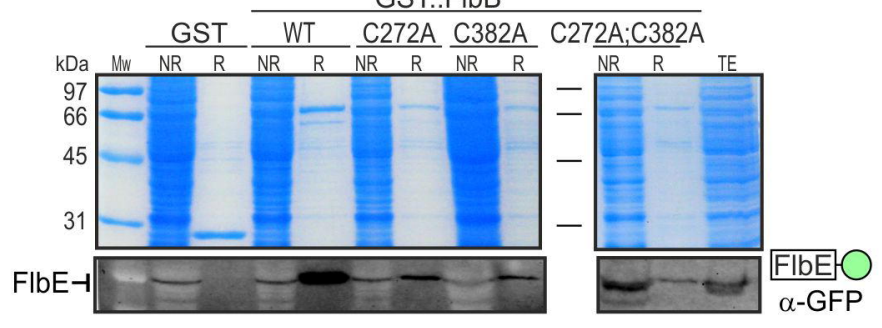

Western-blot Vs coomassie

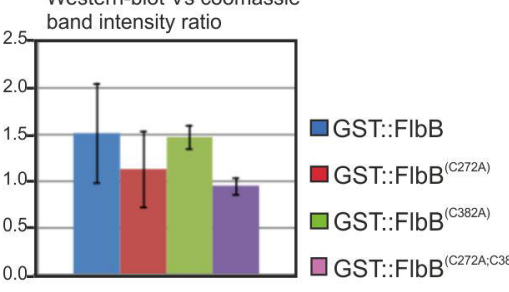


$A_{\text {OEOFEB }} \quad B$
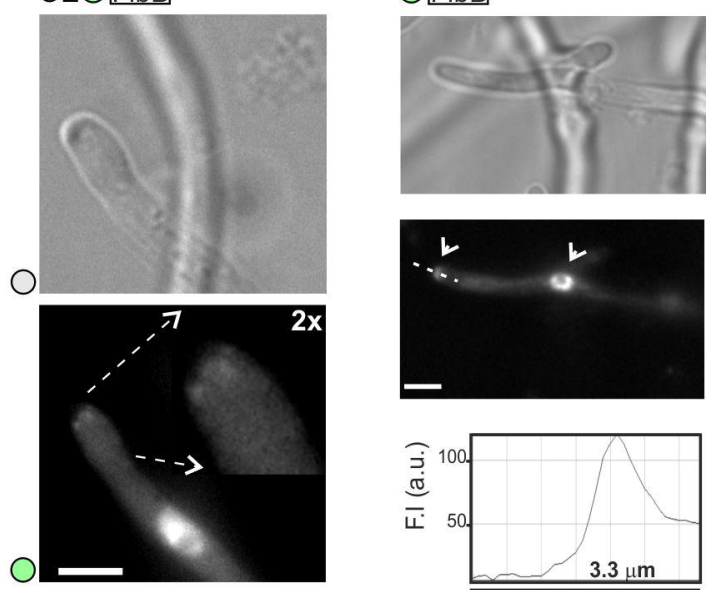

$\triangle m y o E$ OFlbB
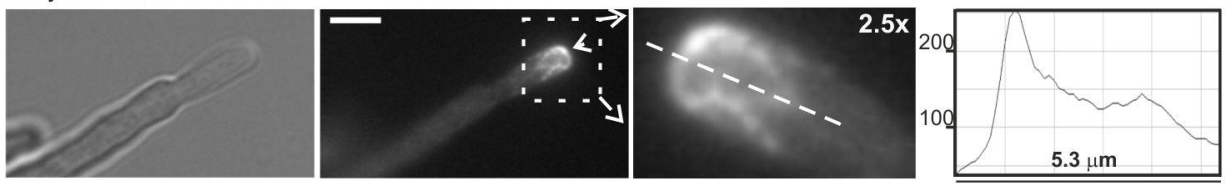

$\triangle m y o E \quad \mathrm{FlbB}$
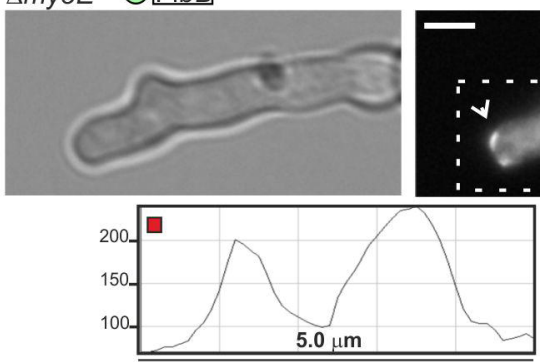
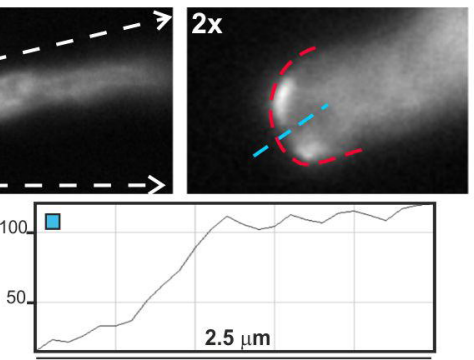

C

OEOFIbB OEFIbEO
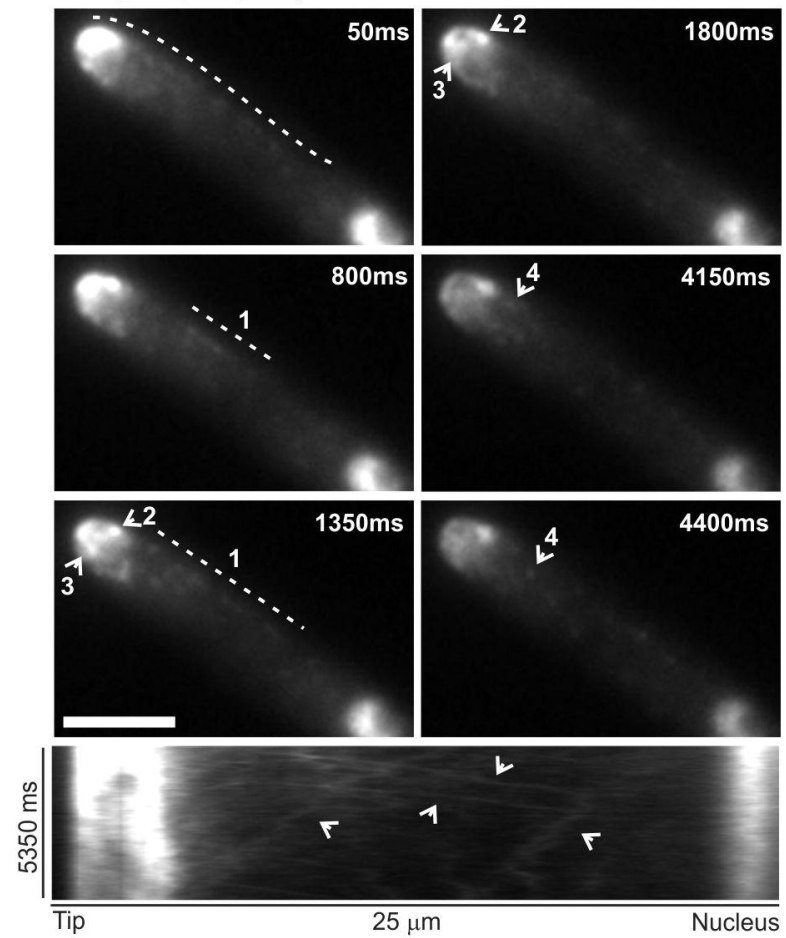

ए

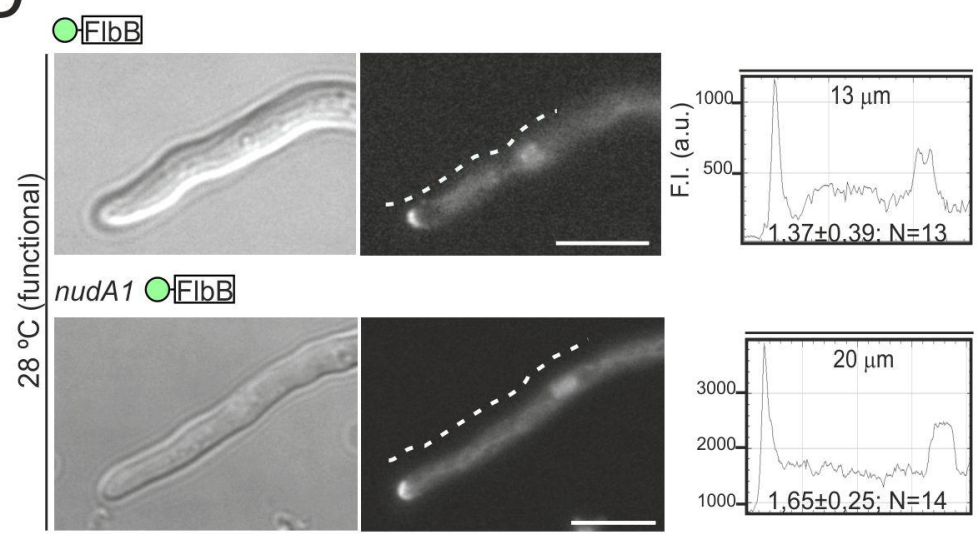

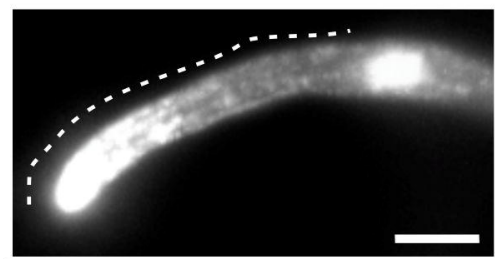
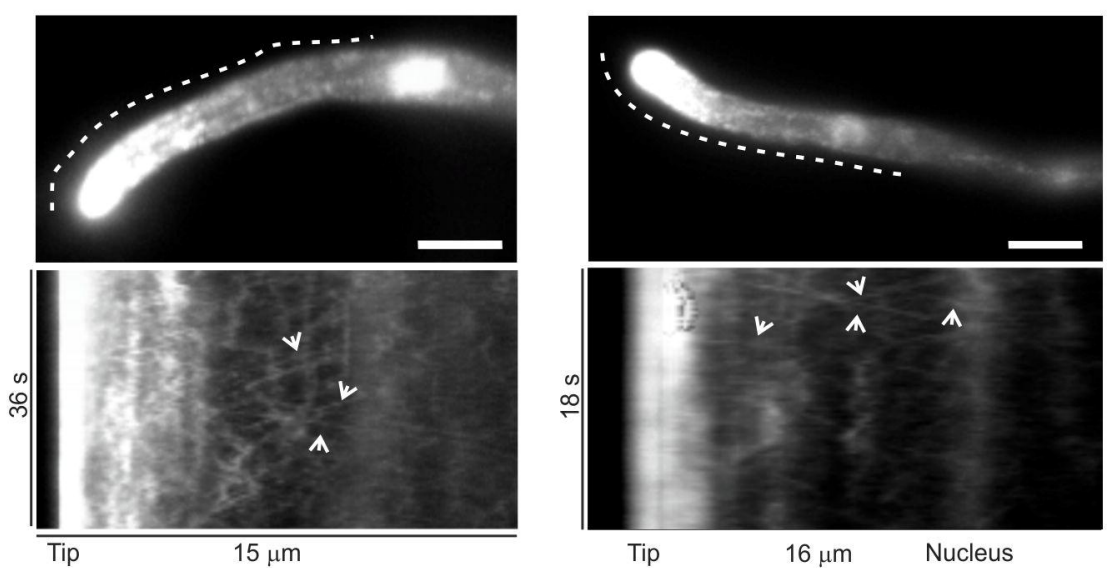

$16 \mu \mathrm{m}$

Nucleus

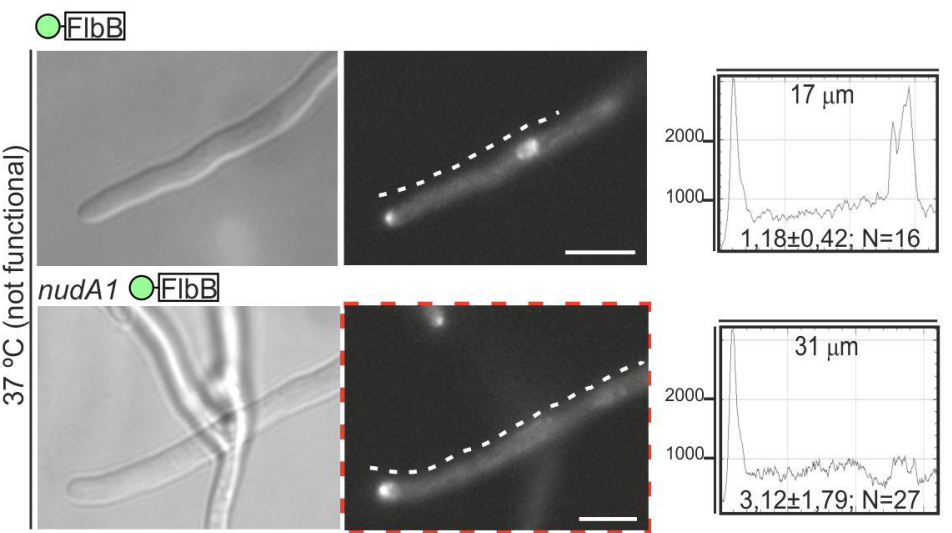




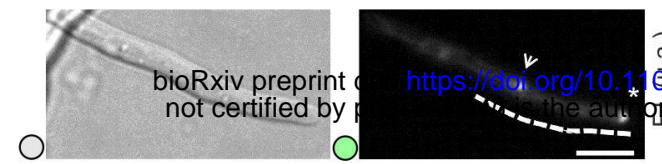

$\triangle f l b D$; FlbB $F[F$
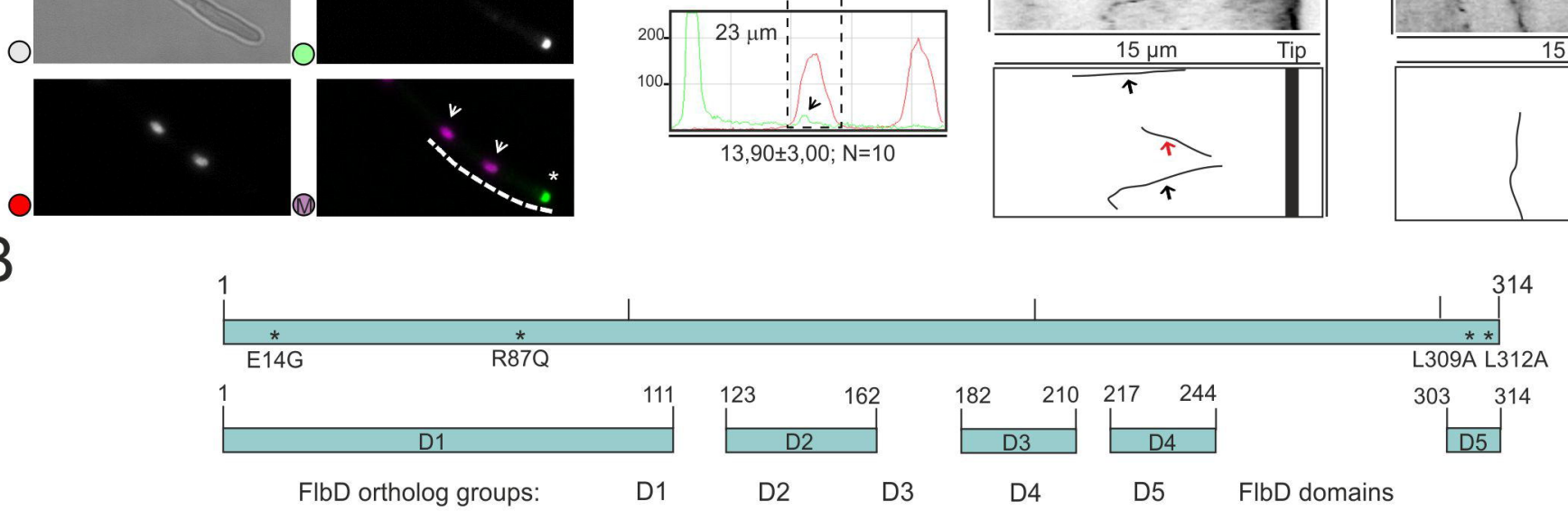

-Eurotiomycetes

\begin{tabular}{rrrr|r}
. Eurotiales..................... & $Y$ & $Y$ & $Y$ & $Y$ \\
. Onygenales..................... & $X$ & $X$ & $Y$ & $Y$ \\
Sordariomycetes.................. & $X$ & $X$ & $X$ & $Y$
\end{tabular}

C
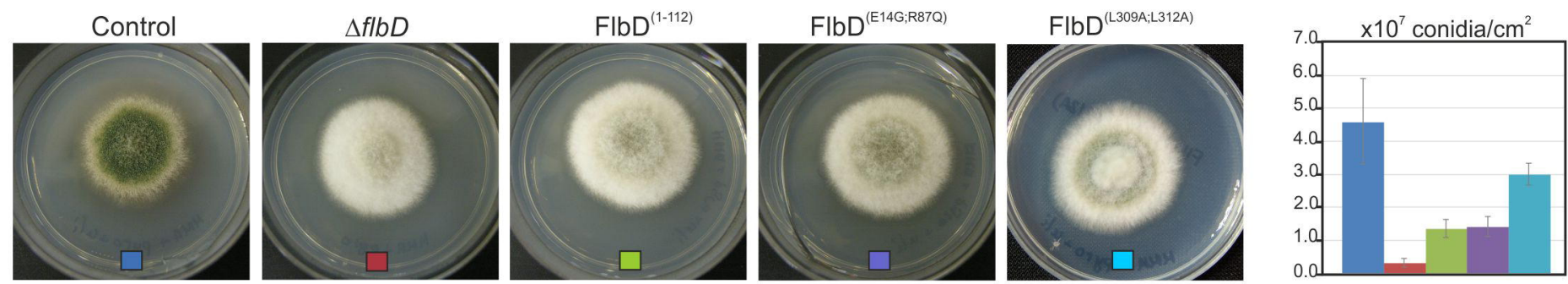

D

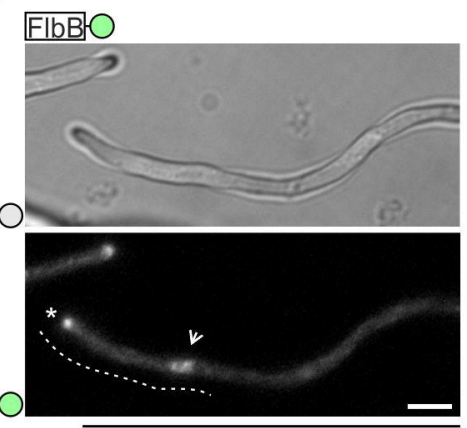

FlbB $O$ FlbD $^{(1-112)}$

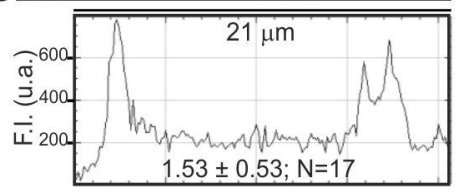

E

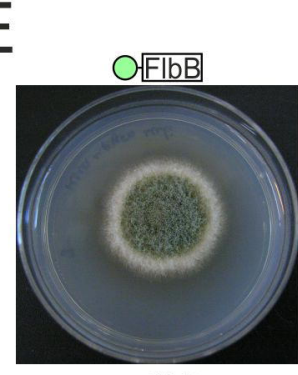

$\triangle f l b B$
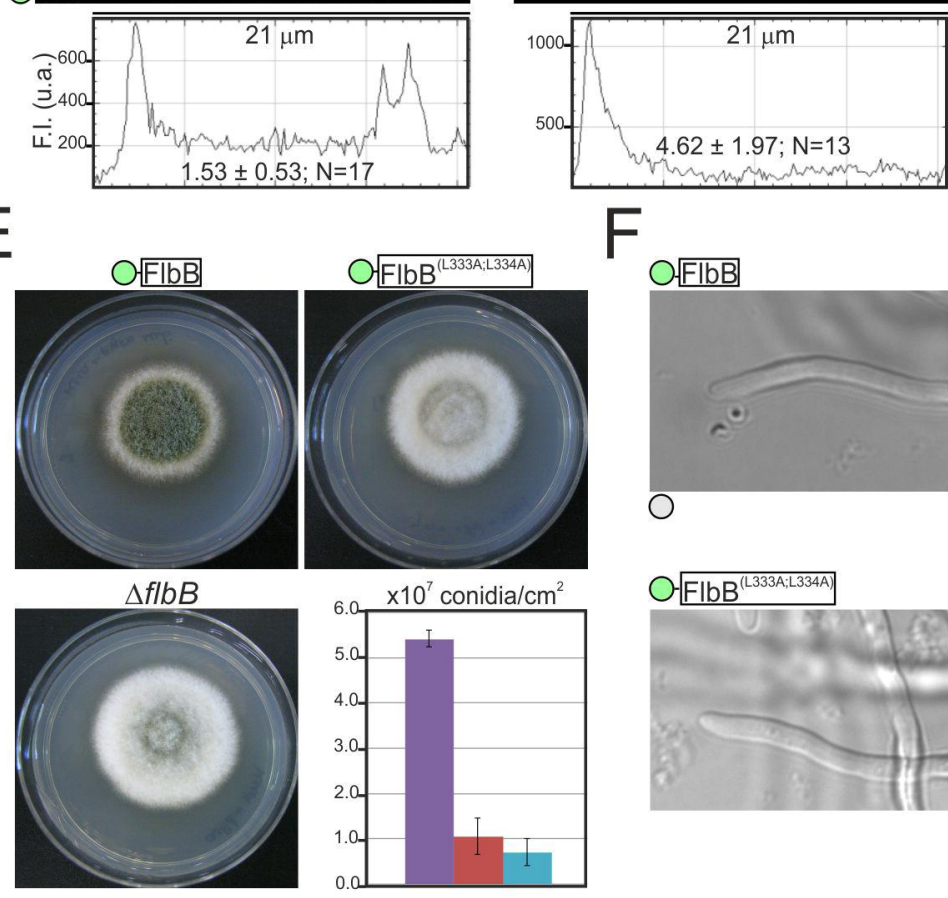

$$
\mathrm{O}
$$

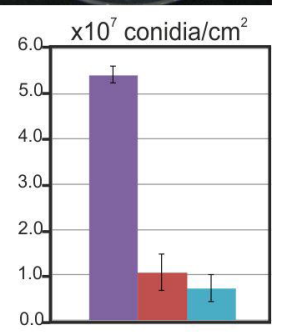

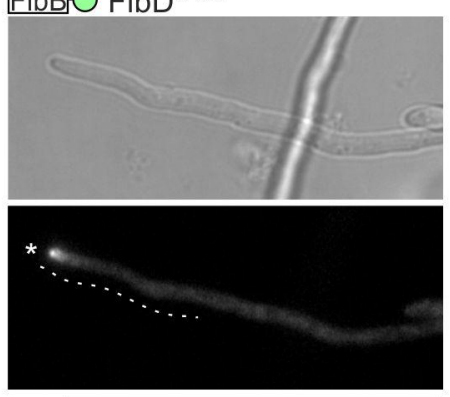

FlbB $O$ FlbD ${ }^{(\text {(E14G;R870) }}$
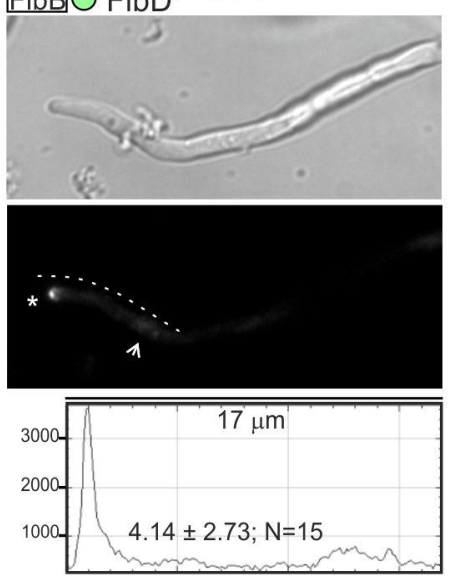

$\mathrm{F}$
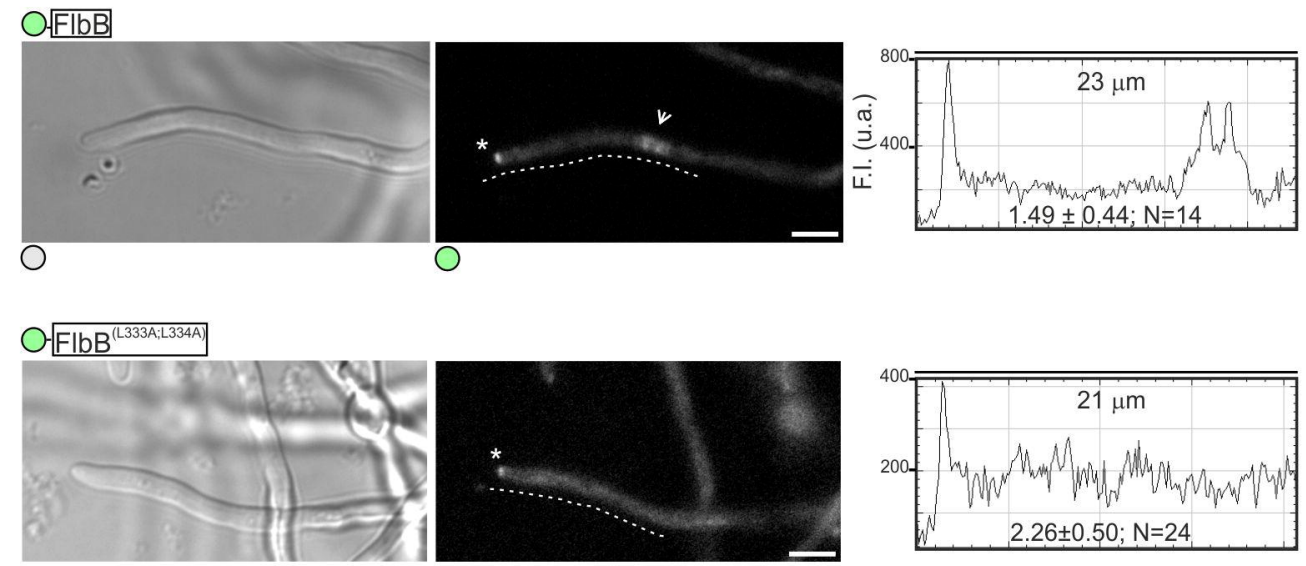

FlbB $O$ FlbD ${ }^{(L 309 A ; L 312 A)}$
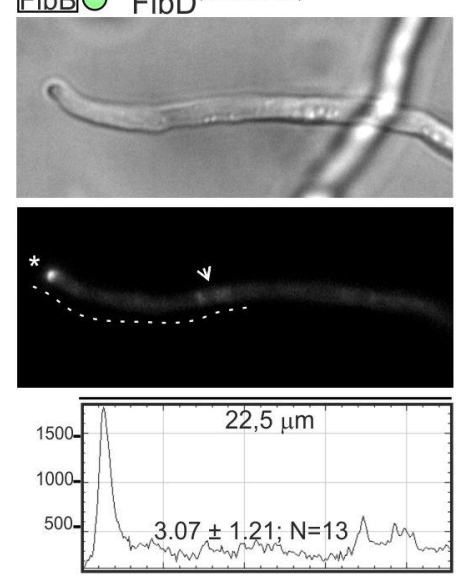

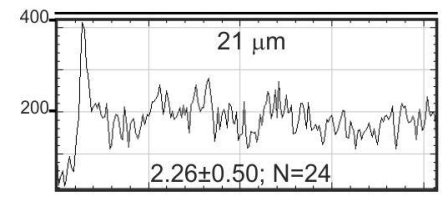




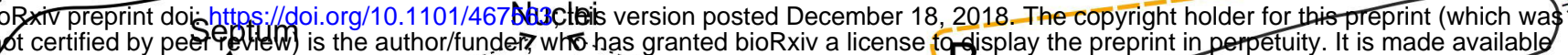
....... under aCC:B $\%=$ NC-ND 4.0 International boense

B

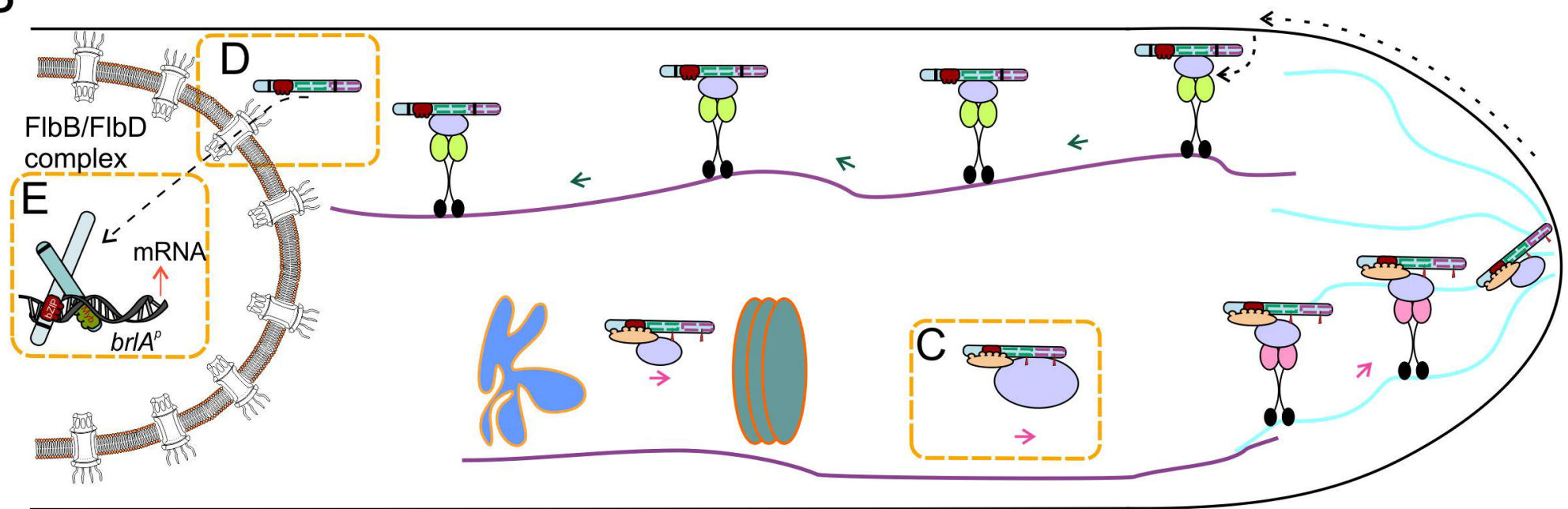

Microtubules

Actin Filaments

$\rightarrow$ Acropetal transport
Endoplamic reticulum

Golgi Cisternae

Vesicle

\section{1:1 Stoichiometry}

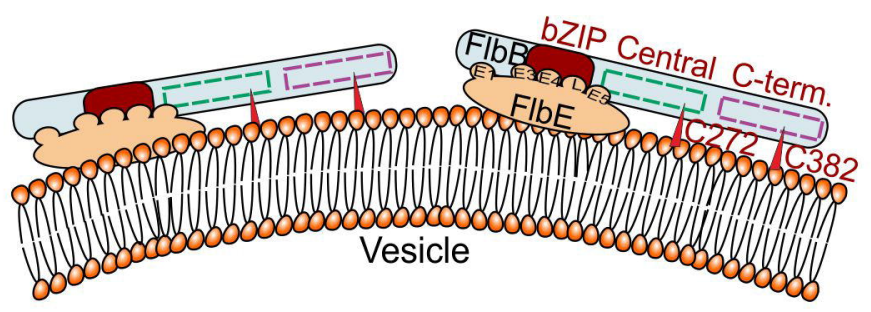

E Heterodimerization

FlbB

bZIP Central C-term.

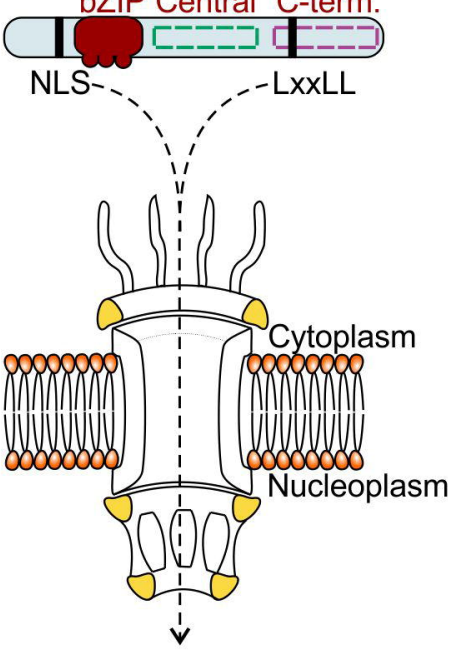

FlbD
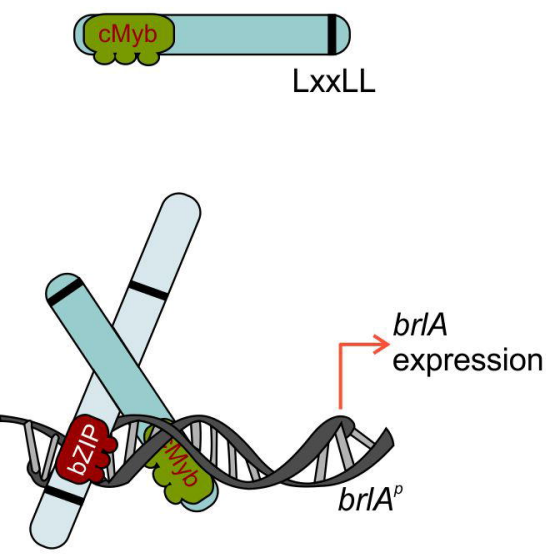

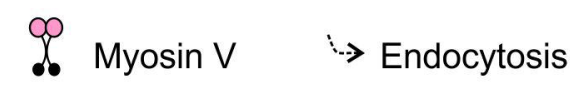

¿o Dynein

$\leftarrow$ Basipetal transport
2:2 Stoichiometry

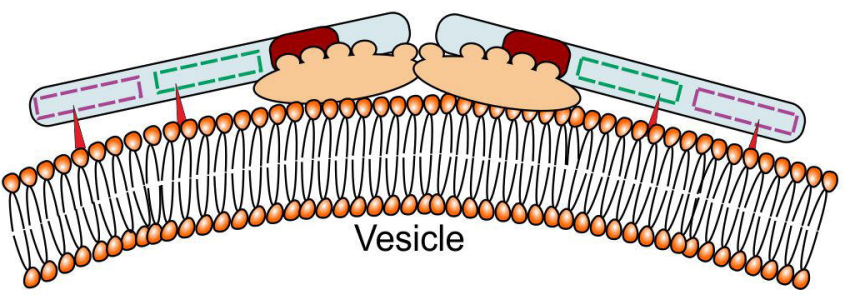

Remodeling + Heterodimerization
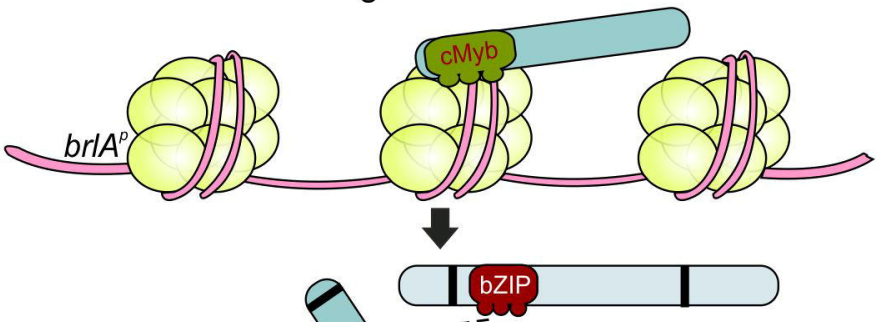

Nuclear pore

FlbD

brlA 


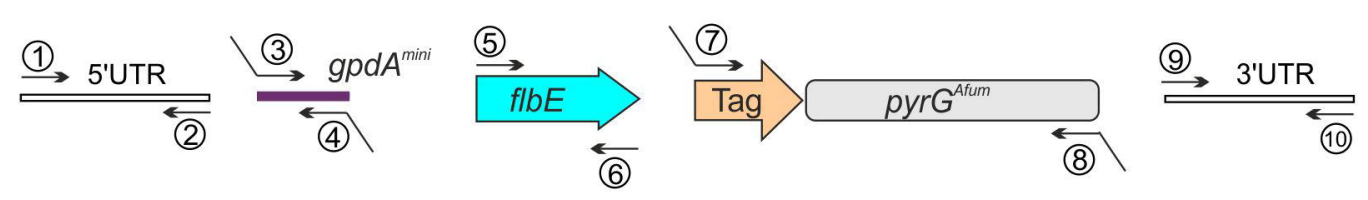

B
(1) flbE-PP1
(2) flbE-PP2-ATG
(3) $g p d A-f l b E-U p$
(4) $g p d A-f l b E-D w$
(5) flbE-geneSP
(6) flbE-GSP2
(7) flbE-GFP1
(8) flbE-GFP2
(9) flbE-GSP3
(10) flbE-GSP4
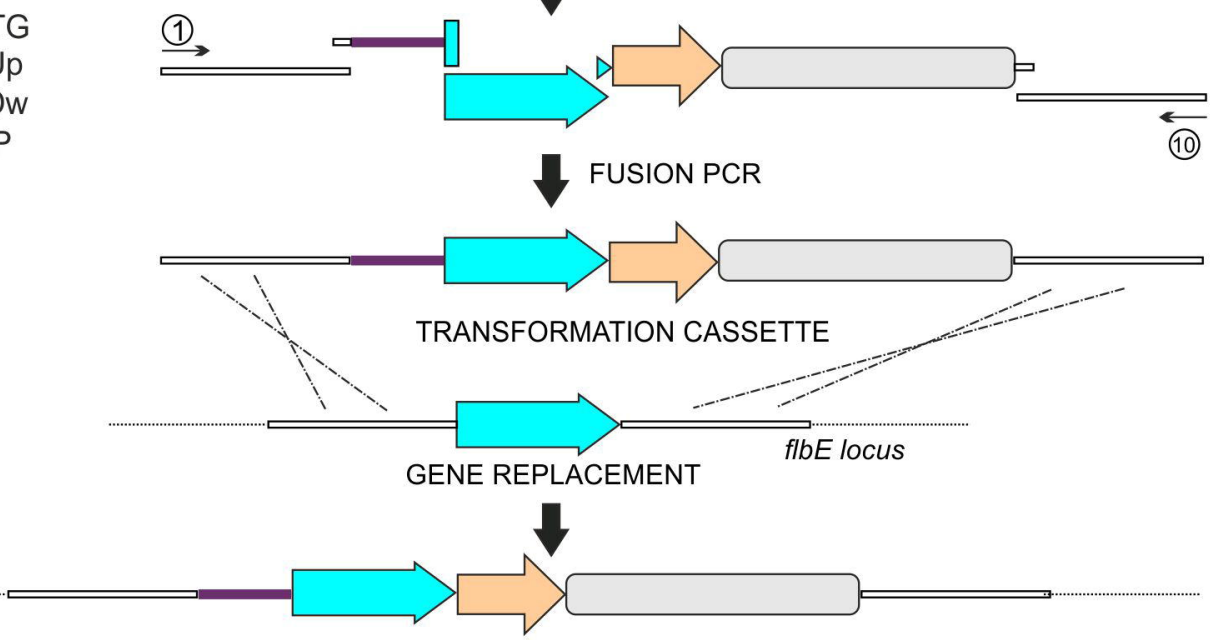

Recombinant tagged flbE locus

C

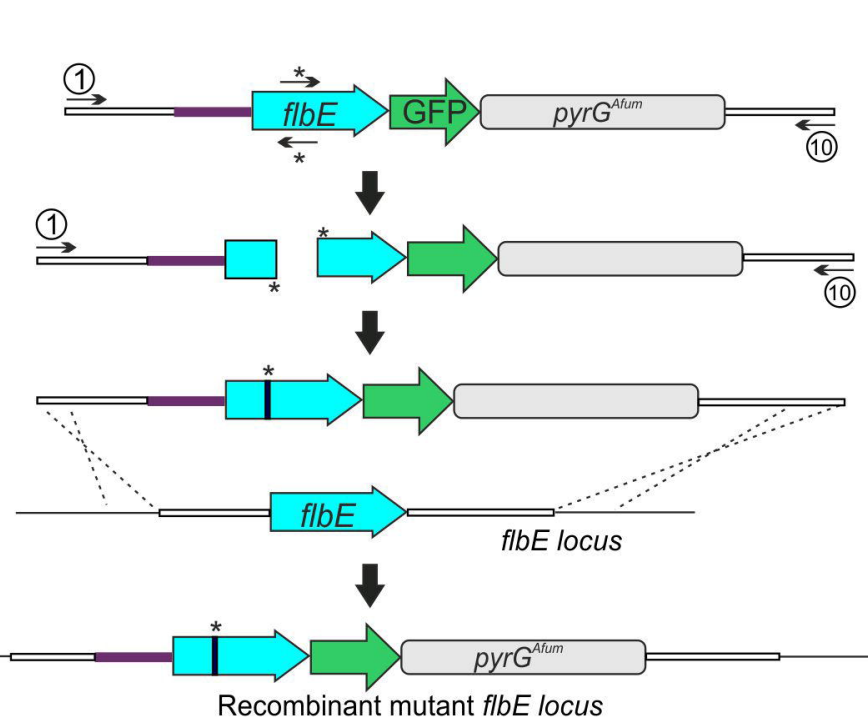

\section{E}
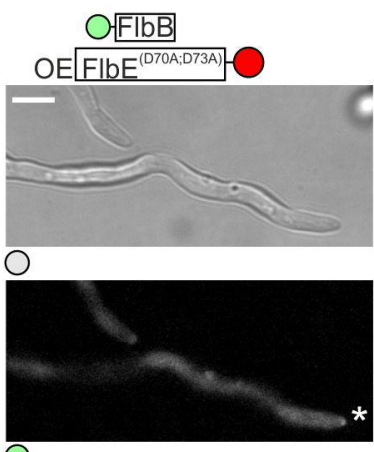

O

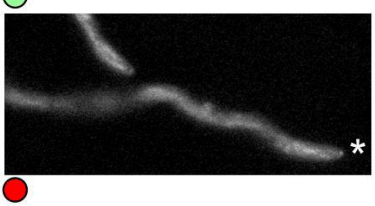

F

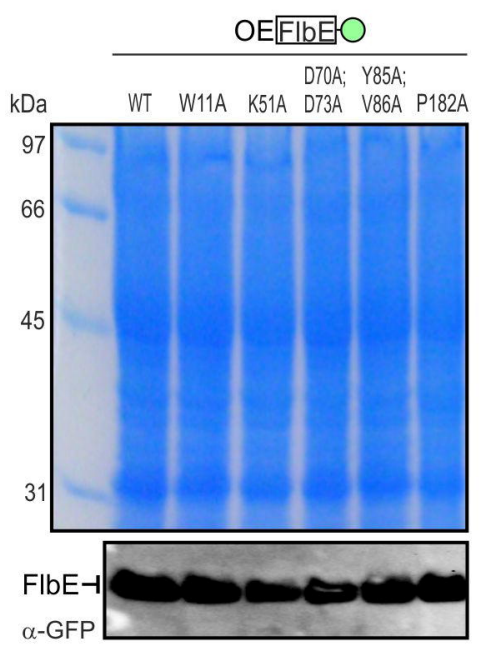

D

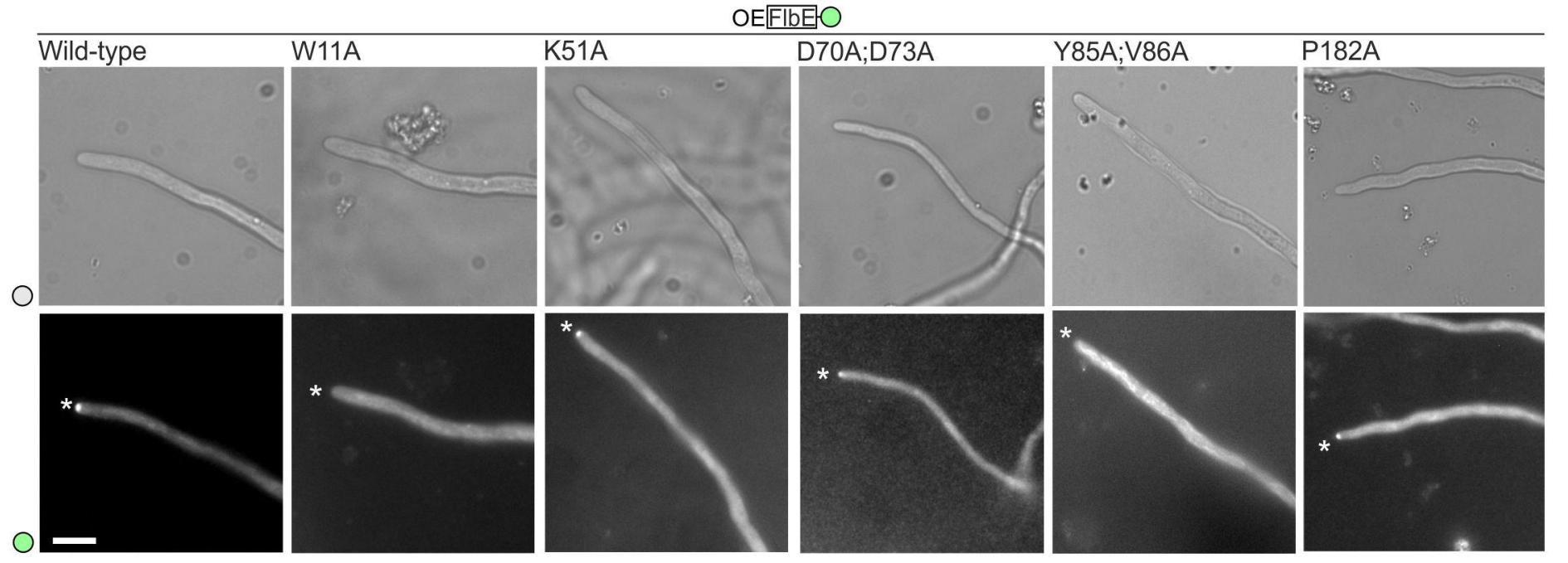



(2) (4)

(1) flbE-PP1

(2) flbE-PP2

(3) flbE-gfpSP

(4) flbE-gfpFP

(5) flbE-geneSP

(6) flbE-GSP4

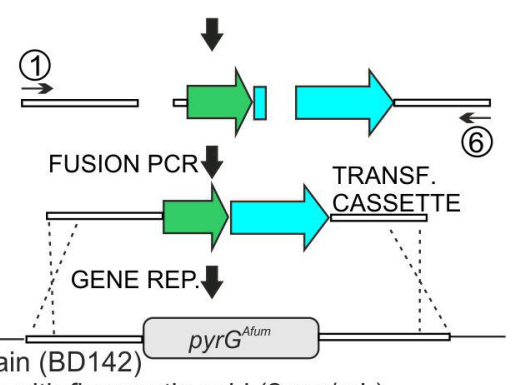

(1) flbE-PP1

(2) flbE-PP2

(3) flbE (ASP)-GSP1-

(4) flbE-gfpFP

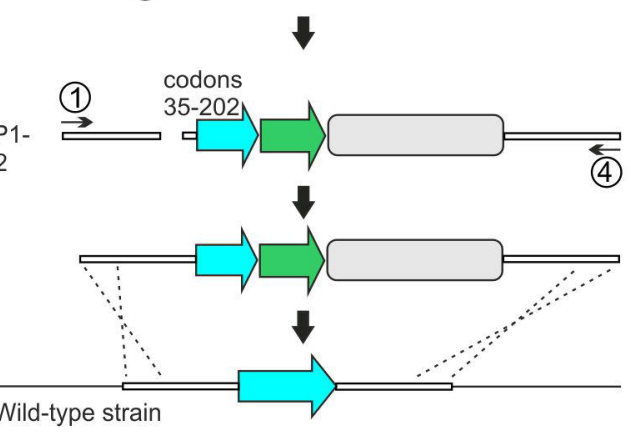

$-\triangle f l b E$ strain (BD142)
-Selection with fluororotic acid $(2 \mathrm{mg} / \mathrm{mL}$ )

-Wild-type strain
(4) $\mathrm{kDa} \mathrm{Pm} \mathrm{NR} \mathrm{R} \mathrm{NR} \mathrm{R}$

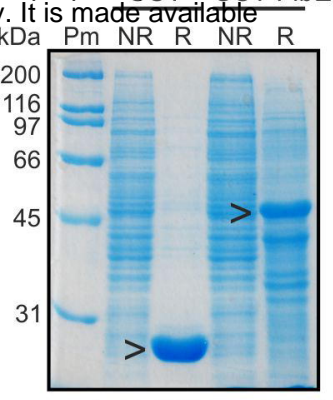

FlbB-

(1) flbE-PP1

(2) flbE-full-forflbB-geneSP

(3) flbB-geneSP

(4) flbB-GFP2

(5) flbE-GSP3 forflbB-GFP2

(6) $\mathrm{flbE}-\mathrm{GSP} 4$
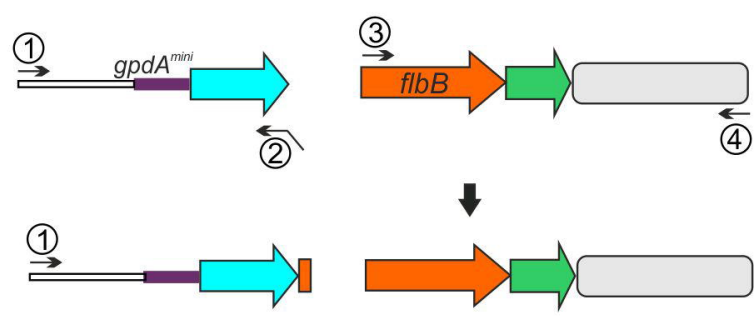

(4)

$\underset{(5)}{\rightarrow}$

(2)
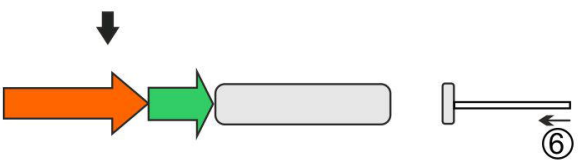

$\checkmark$

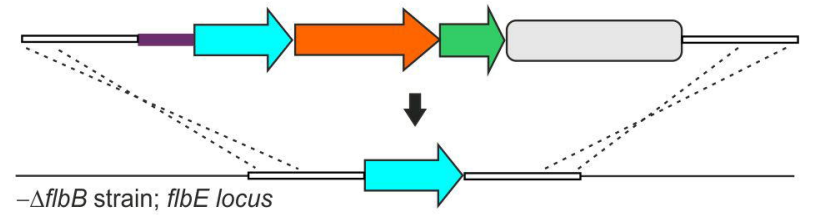

E

(1) flbE-PP1

(2) flbE-PSfor flbB-geneSP

(3) flbB-genesP

(4) flbB-GFP2

(5) flbE-GSP3 forflbB-GFP2

6) flbE-GSP4

(A)

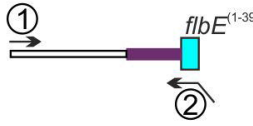

(B) 1<smiles>C=CC(C)(C)C</smiles><smiles>c1ccccc1</smiles>

(C)
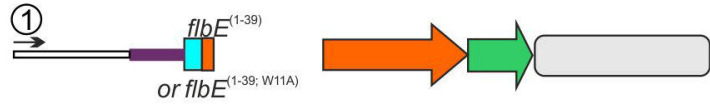

(A)+(C) + (D) $\backslash$ (B) + (C) +(D)
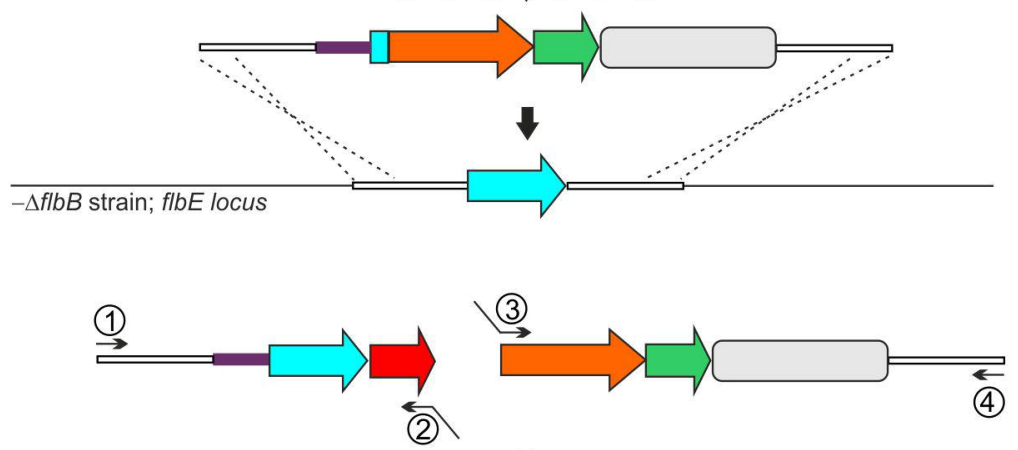

(C)
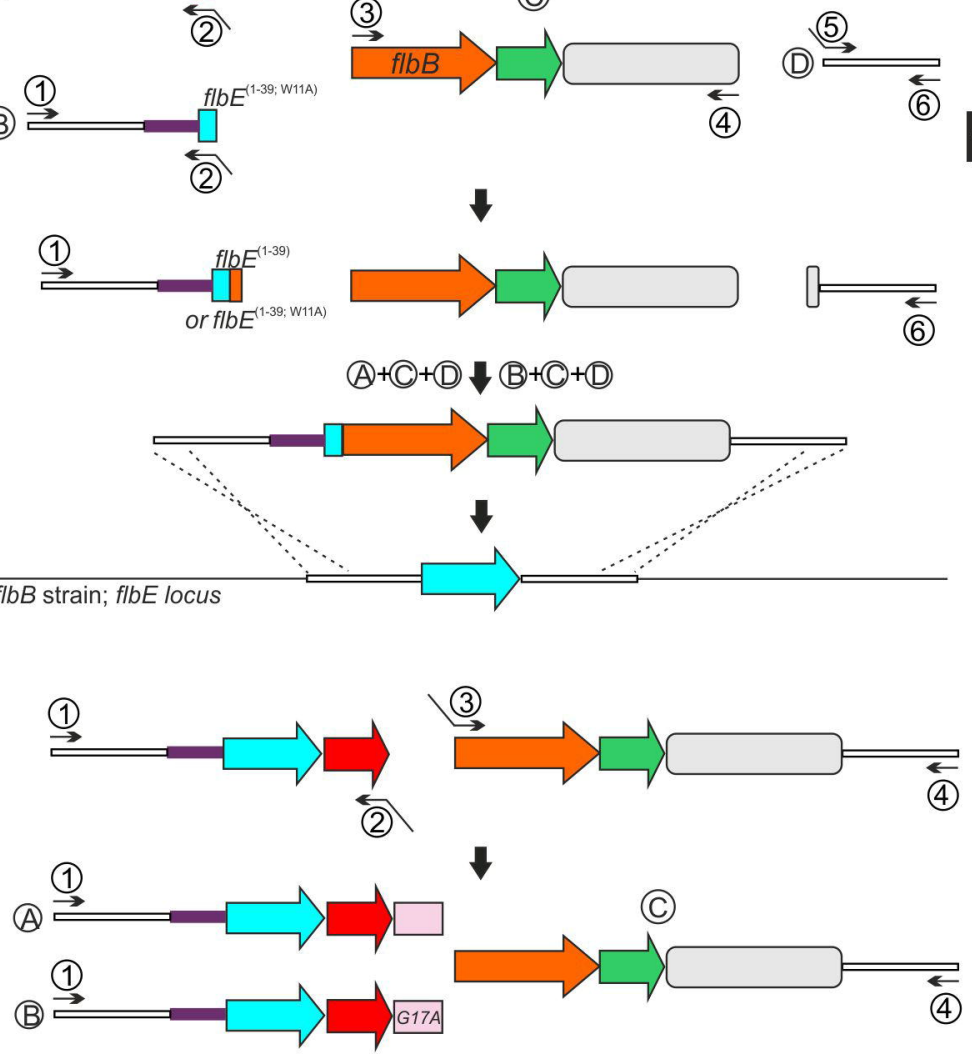

F

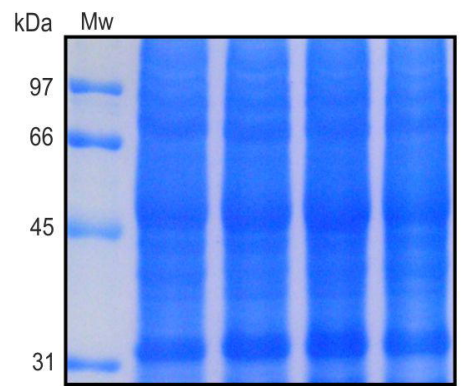

(1) flbE-PP1

(A) + (C) $\vee(B)+C$

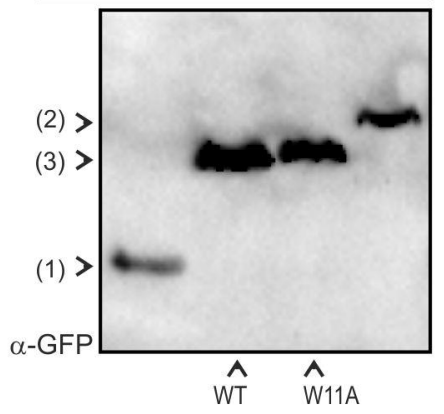

$\mathrm{H}$

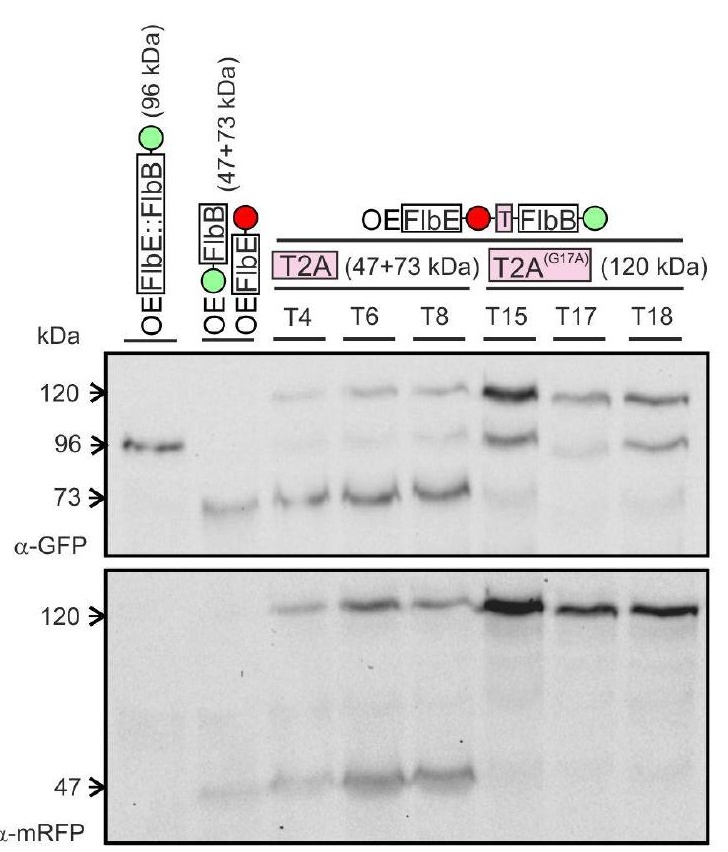

(2) RFP-for-T2A or RFP-for-T2A ${ }^{(G 17 A)}$

(3) T2A-for-flbBgeneSP or ${ }^{\text {T2A }} \mathrm{A}^{(\mathrm{G} 17 \mathrm{~A})}$-for-flbBgeneSP

(4) flbE-GSP4

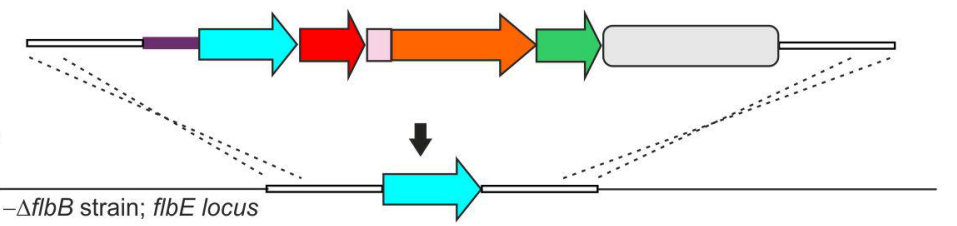



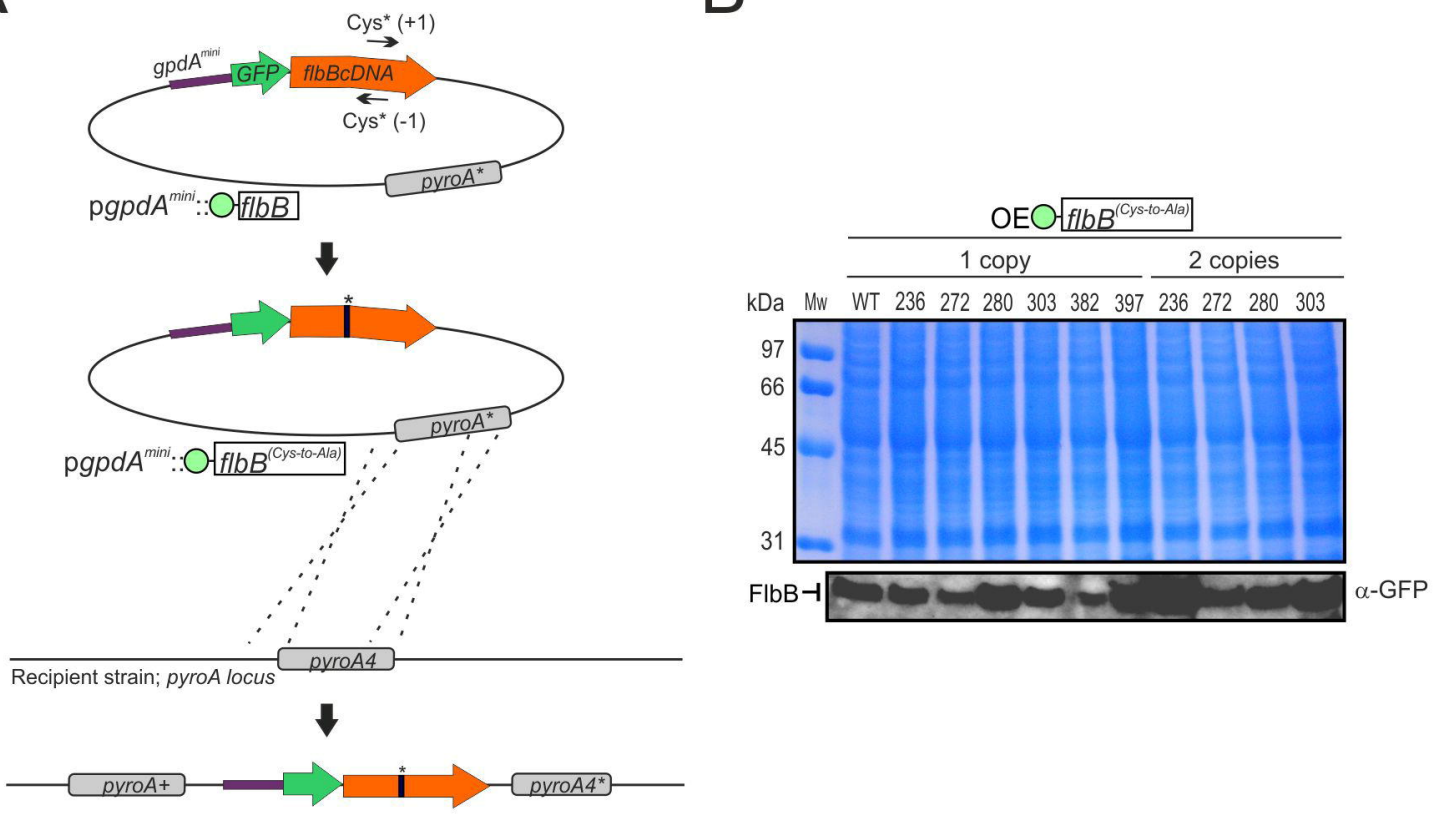

Selection based on a pyroA+ phenotype 
